\title{
Hybrid Invariant Manifolds in Systems with Impulse Effects with Application to Periodic Locomotion in Bipedal Robots
}

\author{
B. Morris* and J.W. Grizzle*
}

\begin{abstract}
Motivated by the problem of controlling walking in a biped with series compliant actuation, this paper develops two main theorems relating to the stabilization of periodic orbits in systems with impulse effects. First, when a periodic orbit of a system with impulse effects lies within a hybrid invariant manifold, the Jacobian linearization of the Poincaré return map results in a matrix that is block upper triangular. One diagonal block is the linearization of the return map of the hybrid zero dynamics, and the other is the product of two sensitivity matrices related to the transverse dynamics. When either sensitivity matrix is sufficiently close to zero, the stability of the return map is determined solely by the hybrid zero dynamics. The second main result of the paper details the construction of a hybrid invariant manifold by introducing impact-updated control parameters. Using the construction, entries of either (or both) of the transverse dynamics' sensitivity matrices can be made arbitrarily small. A simulation example is provided, where stable walking is achieved in a 5-link biped with series compliant actuation.
\end{abstract}

Keywords: hybrid systems; zero dynamics; bipedal robots; underactuated systems.

\section{INTRODUCTION}

The results of this paper are motivated by the study of periodic walking (and running) motions in bipedal robots. The first problem studied concerns a set of sufficient conditions for determining if a periodic solution of an autonomous closed-loop system consisting of a bipedal robot, ground contact model, and feedback controller is exponentially stable. The problem is first cast in terms

${ }^{*}$ Manuscript received February 23, 2007. This work was supported by NSF grant ECS-0600869. B. Morris and J.W. Grizzle are with the Control Systems Laboratory, EECS Department, University of Michigan, Ann Arbor, Michigan 48109-2122, USA. Email: \{morrisbj, grizzle\}@umich.edu Phone: +1 (734) 763-8041 Fax: +1 (734) 763-8041 
of periodic orbits in nonlinear systems with impulse effects [5], [38], that is, systems modeled by an ordinary, time-invariant differential equation (ODE), a co-dimension one switching surface, and a re-initialization rule. Such models can be used to represent a wide range of systems with discontinuous or jump phenomena, including walking and running gaits in legged robots [16], [11]. The method of Poincaré sections is the proper theoretical tool for analyzing the stability of periodic orbits in systems with impulse effects [16], [26], just as it is for ordinary differential equations.

When the method of Poincaré sections is applied in practical problems, it is very common to see the Jacobian linearization of the Poincare map estimated numerically and the exponential stability of a fixed point (i.e., a periodic orbit) deduced on the basis of the eigenvalues. This can be very unwieldy when stability needs to be evaluated repeatedly as part of an iterative procedure to design a feedback controller. Here, the method of Poincaré sections is augmented with notions of (hybrid) invariance, attractivity, and time-scale separation in order to simplify its application to nonlinear systems with impulse effects. The experience gained in [34] in the context of bipedal robots has proven that when stability analysis can be rendered sufficiently tractable, it becomes possible to efficiently explore a large set of asymptotically-stable orbits in order to find one that meets additional performance objectives, such as minimum energy consumption or minimum peak-actuator power demand. The analytical results available in [16] require that an invariant manifold of the ODE portion of a system with impulse effects be rendered finite-time attractive through a continuous, but not Lipschitz continuous, feedback [7]. The result established in this paper will weaken this requirement to attractivity at a sufficiently-rapid exponential rate, thereby permitting the use of smooth feedback laws.

The second main result of the paper is a constructive method for creating the hybrid invariant manifold required by the first result. Hybrid invariance in a nonlinear system with impulse effects refers to a manifold being invariant under both the continuous (ODE) portion of the model as well as the discrete (reset) map present in the model. Invariance in ordinary differential equations is a rich, well-studied subject, and in particular, the methods developed by Byrnes and Isidori for the zero dynamics are well suited for use here. The less-well-studied and hence more challenging problem is how to achieve invariance under the discrete portion of the model. A novel type of dynamic extension of a system with impulse effects is developed for this purpose. The end result is a truly hybrid controller for achieving invariance in a system with impulse effects. 


\section{TECHNICAL BACKGROUND}

This section reviews the definition of a system with impulse effects and introduces the two primary analysis tools that are used in this paper: the method of Poincaré sections and the notion of a hybrid invariant manifold.

The method of Poincaré sections and return maps is widely used to determine the existence and stability of periodic orbits in a broad range of system models, such as time-invariant and periodically-time-varying ordinary differential equations [27], [18], hybrid systems consisting of several time-invariant ordinary differential equations linked by event-based switching mechanisms and re-initialization rules [16], [26], [29], differential-algebraic equations [19], and relay systems with hysteresis [15], to name just a few. While the analytical details may vary significantly from one class of models to another, on a conceptual level, the method of Poincaré is consistent and straightforward: sample the solution of a system according to an event-based or time-based rule, and then evaluate the stability properties of equilibrium points (also called fixed points) of the sampled system, which is called the Poincaré return map; see Fig. 1. Fixed points of the Poincaré map correspond to periodic orbits (limit cycles) of the underlying system.

\section{A. Systems with Impulse Effects}

To define a $C^{1}$ control system with impulse effects, consider a nonlinear affine control system

$$
\dot{x}=f(x)+g(x) u
$$

where the state manifold $\mathcal{X}$ is an open connected subset of $\mathbb{R}^{n}$, the control input $u$ takes values in $\mathcal{U} \subset \mathbb{R}^{m}$, and $f$ and the columns of $g$ are $C^{1}$ vector fields on $\mathcal{X}$. An impact (or switching) surface, $\mathcal{S}$, is a co-dimension one $C^{1}$ submanifold with $\mathcal{S}=\left\{x \in \mathcal{X} \mid H(x)=0, H_{0}(x)>0\right\}$ where $H_{0}: \mathcal{X} \rightarrow \mathbb{R}$ is continuous, $H: \mathcal{X} \rightarrow \mathbb{R}$ is $C^{1}, \mathcal{S} \neq \emptyset$, and $\forall x \in \mathcal{S}, \frac{\partial H}{\partial x}(x) \neq 0$. An impact (or reset) map is a $C^{1}$ function $\Delta: \mathcal{S} \times \mathcal{V} \rightarrow \mathcal{X}, \mathcal{V} \subset \mathbb{R}^{p}, p \geq 0$ where $\mathcal{S} \cap \Delta(\mathcal{S} \times \mathcal{V})=\emptyset$, that is, where the image of the impact map is disjoint from its domain. A $C^{1}$ control system with impulse effects has the form

$$
\Sigma^{o l}:\left\{\begin{array}{rlrl}
\dot{x} & =f(x)+g(x) u & & x^{-} \notin \mathcal{S} \\
x^{+}=\Delta\left(x^{-}, v\right) & & x^{-} \in \mathcal{S}
\end{array}\right.
$$


where $v \in \mathcal{V}$ is a control input for the impact map, and $x^{-}(t)=\lim _{\tau / t} x(\tau)$ and $x^{+}(t)=\lim _{\tau \backslash t} x(\tau)$ are, in words, the left and right limits of a trajectory, $x(t)$. A system with inputs into the vector field but not into the impact map,

$$
\Sigma^{o l}:\left\{\begin{array}{rlrl}
\dot{x} & =f(x)+g(x) u & & x^{-} \notin \mathcal{S} \\
x^{+}=\Delta\left(x^{-}\right) & & x^{-} \in \mathcal{S}
\end{array},\right.
$$

can be written as a special case of (2) with $\mathcal{V}=\emptyset$. Replacing the control system (1) with an autonomous system

$$
\dot{x}=f(x),
$$

and taking $\mathcal{V}=\emptyset$ leads to a $C^{1}$ autonomous system with impulse effects,

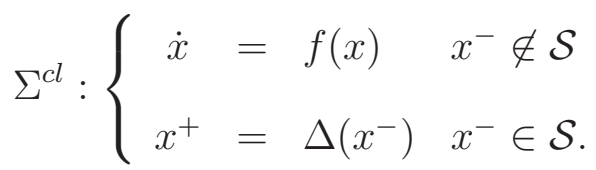

For compactness of notation, an autonomous system with impulse effects (5) will be denoted as a 4-tuple, $\Sigma^{c l}=(\mathcal{X}, \mathcal{S}, \Delta, f)$, while a control system with impulse effects (2) will be denoted as a 7-tuple, $\Sigma^{o l}=(\mathcal{X}, \mathcal{S}, \mathcal{V}, \mathcal{U}, \Delta, f, g)$.

In simple terms, a solution of (2) or (5) is specified by the differential equation (1) or (4) until its state "impacts" the hyper surface $\mathcal{S}$ at some time $t_{I}$. At $t_{I}$, the impact model $\Delta$ compresses the impact event into an instantaneous moment of time, resulting in a discontinuity in the state trajectory. The impact model provides the new initial condition from which the differential equation evolves until the next impact with $\mathcal{S}$. In order to avoid the state having to take on two values at the "impact time" $t_{I}$, the impact event is, roughly speaking, described in terms of the values of the state "just prior to impact" at time " $t_{I}^{-}$", and "just after impact" at time " $t_{I}^{+}$". These values are represented by $x^{-}$and $x^{+}$, respectively. From this description, a formal definition of a solution is written down by piecing together appropriately initialized solutions of (1) or (4); see [38], [16], [26], [10]. A choice must be made whether the solution of (5) is a left- or a right-continuous function of time at each impact event; here, solutions are assumed to be right continuous [16].

\section{B. Periodic Orbits and the Poincaré Return Map}

Cyclic behaviors such as walking are represented as periodic orbits of systems with impulse effects. A solution $\varphi\left(t, t_{0}, x_{0}\right)$ of an autonomous system $\Sigma^{c l}$ is periodic if there exists a finite 
$T>0$ such that $\varphi\left(t+T, t_{0}, x_{0}\right)=\varphi\left(t, t_{0}, x_{0}\right)$ for all $t \in\left[t_{0}, \infty\right)$. A set $\mathcal{O} \subset \mathcal{X}$ is a periodic orbit if $\mathcal{O}=\left\{\varphi\left(t, t_{0}, x_{0}\right) \mid t \geq t_{0}\right\}$ for some periodic solution $\varphi\left(t, t_{0}, x_{0}\right)$. If a periodic solution has an impact event, then the corresponding periodic orbit $\mathcal{O}$ is not closed; see [16], [24]. Let $\overline{\mathcal{O}}$ denote its set closure. Given a norm $\|\cdot\|$ on $\mathcal{X}$, the distance from a point $x \in \mathcal{X}$ to a set $F \subset \mathcal{X}$ is defined as $\operatorname{dist}(x, F)=\inf _{\bar{x} \in F}\|x-\bar{x}\|$. Notions of stability in the sense of Lyapunov, asymptotic stability, and exponential stability of orbits follow the standard definitions as in [23, pp. 302], [16], [26]. In a similar manner, a periodic orbit of the control system $\Sigma^{o l}$ is defined as the set of points in $\mathcal{X}$ traced out by a periodic solution of $\Sigma^{o l}$ for some inputs $u$ and $v$.

When using the method of Poincaré to study systems with impulse effects, it is natural to select $\mathcal{S}$ as the Poincaré section. To define the return map, let $\phi\left(t, x_{0}\right)$ be the maximal solution of (4) with initial condition $x_{0}$ at time $t_{0}=0$. The time-to-impact function, $T_{I}(x)$, is the time from initialization to the first intersection with the set $\mathcal{S}$ and is in general a partial map. The Poincaré return map, $P: \mathcal{S} \rightarrow \mathcal{S}$, is then the partial map $P(x)=\phi\left(T_{I} \circ \Delta(x), \Delta(x)\right)$. A periodic orbit $\mathcal{O}$ has period one ${ }^{1}$ if its closure intersects $\mathcal{S}$ at exactly one point, $x^{*}=\overline{\mathcal{O}} \cap \mathcal{S}$. A period one orbit is transversal to $\mathcal{S}$ if $L_{f} H\left(x^{*}\right)=\frac{\partial H}{\partial x}\left(x^{*}\right) f\left(x^{*}\right) \neq 0$ (in words, the vector field $f$ is not tangent to $\mathcal{S}$ at the point $\left.x^{*}\right)$. For convenience, define the partial function $\phi_{T_{I}}(x)=\phi\left(T_{I}(x), x\right)$ so that the Poincaré return map can be written as $P(x)=\phi_{T_{I}} \circ \Delta(x)$. For the case of autonomous systems with impulse effects, the Method of Poincaré sections is formalized in the following theorem:

Theorem 0 (Method of Poincaré Sections): If the $C^{1}$ autonomous system with impulse effects $\Sigma^{c l}=(\mathcal{X}, \mathcal{S}, \Delta, f)$ has a periodic orbit $\mathcal{O}$ that is transversal to $\mathcal{S}$, then the following are equivalent:

i) $x^{*}$ is an exponentially stable (resp., asymp. stable, or stable i.s.L.) fixed point of $P$;

ii) $\mathcal{O}$ is an exponentially stable (resp., asymp. stable, or stable i.s.L.) periodic orbit.

Proof: The proof for exponential stability is in [24]. Proofs for asymptotic stability and stability i.s.L are in [16], [26].

\footnotetext{
${ }^{1}$ Unless otherwise stated, all periodic orbits are assumed to have period one.
} 


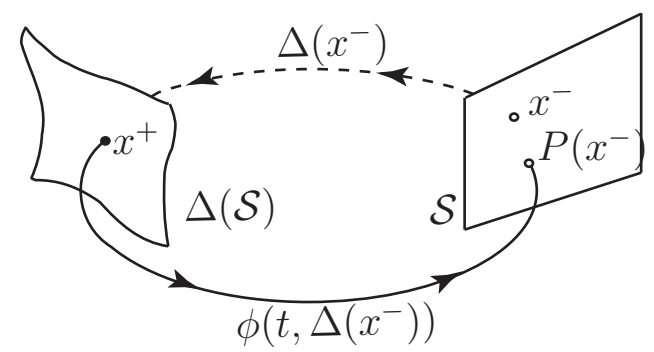

Fig. 1. Geometric interpretation of a Poincaré return map $P: \mathcal{S} \rightarrow \mathcal{S}$ for a system with impulse effects. The Poincaré section is selected as the switching surface, $\mathcal{S}$. A periodic orbit exists when $P\left(x^{-}\right)=x^{-}$. Due to right-continuity of the solutions, $x^{-}$ is not an element of the orbit. With left-continuous solutions, $\Delta\left(x^{-}\right)$would not be an element of the orbit.

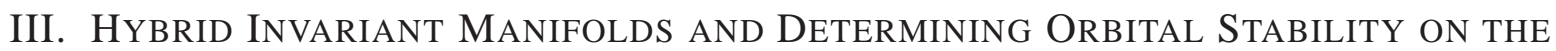

\section{BASIS OF A RESTRICTION DYNAMICS}

This section identifies properties of the autonomous system (5) under which the exponential stabilizability of a periodic orbit can be determined on the basis of a hybrid restriction dynamics. The key hypothesis will be the existence of an embedded submanifold that is invariant under both the continuous and discrete portions of the hybrid model (5). The design of static and dynamic state variable feedbacks that create invariant submanifolds for systems modeled by ordinary differential equations is a well-studied problem and plays a prominent role in the notion of the zero dynamics [21]. How to design feedbacks that achieve invariance under the impact map in (2) is the topic of Section IV.

\section{A. Hybrid Invariance and Restriction Dynamics}

For an autonomous system with impulse effects $\Sigma^{c l}=(\mathcal{X}, \mathcal{S}, \Delta, f)$, a submanifold $\mathcal{Z} \subset \mathcal{X}$ is forward invariant if for each point $x$ in $\mathcal{Z}, f(x) \in T_{x} \mathcal{Z}$. A submanifold $\mathcal{Z}$ is impact invariant in an autonomous system with impulse effects $\Sigma^{c l}=(\mathcal{X}, \mathcal{S}, \Delta, f)$ or in a control system with impulse effects $\Sigma^{o l}=(\mathcal{X}, \mathcal{S}, \emptyset, \mathcal{U}, \Delta, f, g)$, if for each point $x$ in $\mathcal{S} \cap \mathcal{Z}, \Delta(x) \in \mathcal{Z}$. A submanifold $\mathcal{Z}$ is hybrid invariant if it is both forward invariant and impact invariant. If a $C^{1}$ embedded submanifold $\mathcal{Z}$ is hybrid invariant and $\mathcal{S} \cap \mathcal{Z}$ is $C^{1}$ with dimension one less than that of $\mathcal{Z}$, then

$$
\left.\Sigma^{c l}\right|_{\mathcal{Z}}:\left\{\begin{array}{rlr}
\dot{z} & =\left.f\right|_{\mathcal{Z}}(z) & z^{-} \notin \mathcal{S} \cap \mathcal{Z} \\
z^{+}=\left.\Delta\right|_{\mathcal{S} \cap \mathcal{Z}}\left(z^{-}\right) & z^{-} \in \mathcal{S} \cap \mathcal{Z}
\end{array}\right.
$$


is called a hybrid restriction dynamics of the autonomous system $\Sigma^{c l}$, where $\left.f\right|_{\mathcal{Z}}$ and $\left.\Delta\right|_{\mathcal{S} \cap \mathcal{Z}}$ are the restrictions of $f$ and $\Delta$ to $\mathcal{Z}$ and $\mathcal{S} \cap \mathcal{Z}$, respectively. If, in addition, the system $\Sigma^{c l}$ has a periodic orbit $\mathcal{O} \subset \mathcal{Z}$, then $\mathcal{O}$ is a periodic orbit of the hybrid restriction dynamics. The system (6) will sometimes be denoted as $\left.\Sigma^{c l}\right|_{\mathcal{Z}}=\left(\mathcal{Z}, \mathcal{S} \cap \mathcal{Z},\left.\Delta\right|_{\mathcal{S} \cap \mathcal{Z}},\left.f\right|_{\mathcal{Z}}\right)$. Hybrid invariance of $\mathcal{Z}$ is reflected in the Poincaré map as

$$
P(\mathcal{S} \cap \mathcal{Z}) \subset \mathcal{S} \cap \mathcal{Z}
$$

On the basis of (7), the restricted Poincaré map, $\rho: \mathcal{S} \cap \mathcal{Z} \rightarrow \mathcal{S} \cap \mathcal{Z}$, is defined as $\rho=\left.P\right|_{\mathcal{Z}}$, or equivalently,

$$
\rho(z)=\left.\phi\right|_{\mathcal{Z}}\left(\left.\left.T_{I}\right|_{\mathcal{Z}} \circ \Delta\right|_{\mathcal{S} \cap \mathcal{Z}}(z),\left.\Delta\right|_{\mathcal{S} \cap \mathcal{Z}}(z)\right)=\left.\left.\phi_{T_{I}}\right|_{\mathcal{Z}} \circ \Delta\right|_{\mathcal{S} \cap \mathcal{Z}}(z)
$$

\section{B. Factoring the Sensitivities of the Transverse Dynamics}

The following theorem identifies conditions under which the exponential stability of a periodic orbit of the restriction dynamics is inherited by the full model. The importance of such conditions in the design of controllers that create stable, periodic locomotion patterns in biped robots has been studied before in [16], [34], where finite-time convergence to an invariant manifold was assumed. The result presented here relaxes this to "sufficiently rapid" exponential convergence. A less general version was first presented in [24], and applied to 3D bipedal locomotion in [3].

Theorem 1: Consider a $C^{1}$ autonomous system with impulse effects $\Sigma^{c l}=(\mathcal{X}, \mathcal{S}, \Delta, f)$ and assume there exists a $C^{1}$ embedded $k$-dimensional submanifold $\mathcal{Z}$ such that

H1.1) $\mathcal{Z}$ is hybrid invariant;

H1.2) $\mathcal{S} \cap \mathcal{Z}$ is a $C^{1}$ embedded submanifold and has dimension one less than $\mathcal{Z}$; and

H1.3) $\Sigma^{c l}$ has a periodic orbit $\mathcal{O}$ transversal to $\mathcal{S}$ and contained in $\mathcal{Z}$.

Then, there exist local changes of coordinates $\Gamma: U \rightarrow \mathbb{R}^{k-1} \times \mathbb{R}^{n-k}$ and $\Psi: V \rightarrow \mathbb{R}^{k} \times \mathbb{R}^{n-k}$, about $x^{*}=\overline{\mathcal{O}} \cap \mathcal{S}$ and $\Delta\left(x^{*}\right)$, respectively, such that when the Poincaré map of the system $\Sigma^{c l}$ is represented in the new coordinates, its Jacobian ${ }^{2}$ about the fixed point $x^{*}$ is

$$
\mathcal{D} \hat{P}\left(z^{*}, \eta^{*}\right)=\left[\begin{array}{c|c}
\mathcal{D} \rho\left(z^{*}\right) & \star \\
\hline 0 & S_{\phi_{T_{I}}}\left(\bar{z}^{*}, \bar{\eta}^{*}\right) S_{\Delta}\left(z^{*}, \eta^{*}\right)
\end{array}\right],
$$

\footnotetext{
${ }^{2}$ For a differentiable function $g\left(x_{1}, x_{2}, \ldots, x_{p}\right)$, the notation $\mathcal{D}_{i} g\left(y_{1}, y_{2}, \ldots, y_{p}\right)$ refers to $\partial g / \partial x_{i}$ evaluated at $\left(x_{1}, x_{2}, \ldots, x_{p}\right)=$ $\left(y_{1}, y_{2}, \ldots, y_{p}\right)$. The argument $x_{i}$ may be a vector. $\mathcal{D} g\left(y_{1}, \ldots, y_{p}\right)$ is $\left(\partial g / \partial x_{1}, \ldots, \partial g / \partial x_{p}\right)$ evaluated at $\left(x_{1}, \ldots, x_{p}\right)=$ $\left(y_{1}, \ldots, y_{p}\right)$.
} 
where $^{3} \hat{P}=\Gamma \circ P \circ \Gamma^{-1}, \rho$ is the restricted map of (8), $S_{\phi_{T_{I}}}\left(\bar{z}^{*}, \bar{\eta}^{*}\right)=\mathcal{D}_{2}\left(\Gamma_{2} \circ \phi_{T_{I}} \circ \Psi^{-1}\right)\left(\bar{z}^{*}, \bar{\eta}^{*}\right)$, and $S_{\Delta}\left(z^{*}, \eta^{*}\right)=\mathcal{D}_{2}\left(\Psi_{2} \circ \Delta \circ \Gamma^{-1}\right)\left(z^{*}, \eta^{*}\right)$, for $\left(z^{*}, \eta^{*}\right)=\left(\Gamma_{1}\left(x^{*}\right), \Gamma_{2}\left(x^{*}\right)\right)=\Gamma\left(x^{*}\right)$ and $\left(\bar{z}^{*}, \bar{\eta}^{*}\right)=\Psi \circ \Delta\left(x^{*}\right)=\left(\Psi_{1} \circ \Delta\left(x^{*}\right), \Psi_{2} \circ \Delta\left(x^{*}\right)\right)$.

The proof is given in the appendix. This theorem identifies two features present in the linearized Poincaré map evaluated at a fixed point lying in a hybrid invariant manifold. The first is the upper-triangular structure, which is immediate from the hybrid invariance of $\mathcal{Z}$; see (7). The second, and more interesting result, is that the bottom right block is the product of $S_{\phi_{T_{I}}}$ and $S_{\Delta}$, which are the sensitivities of the transverse dynamics with respect to the continuous flow and impact map, respectively. If either of the sensitivities $S_{\phi_{T_{I}}}$ or $S_{\Delta}$ can be made sufficiently small, then the spectral radius of $\mathcal{D} \hat{P}$ will be determined solely by the restricted Poincaré map. Corollary 2 below is based on the observation that $S_{\phi_{T_{I}}}$ can be made small through sufficiently rapid convergence of the transversal dynamics to $\mathcal{Z}$. The next section will address $S_{\Delta}$ showing that this term can be made arbitrarily small by controlling the behavior of the impact map.

Corollary 2: Consider a family of $C^{1}$ autonomous systems with impulse effects with the vector field of each member depending on a real parameter $\epsilon>0, \Sigma^{c l, \epsilon}=\left(\mathcal{X}, \mathcal{S}, \Delta, f^{\epsilon}\right)$. Assume that for each value of $\epsilon \in(0, \infty)$, Hypotheses H1.1) - H1.3) are met and also that

H2.1) the submanifold $\mathcal{Z}$ and fixed point $x^{*}$ are independent of $\epsilon$;

H2.2) $f^{\epsilon}$ restricted to $\mathcal{Z}$ is independent of $\epsilon$, so that $\left.f\right|_{\mathcal{Z}}=\left.f^{\epsilon}\right|_{\mathcal{Z}}$ for any $\epsilon \in(0, \infty)$; and

H2.3) there exists a function $K:(0, \infty) \rightarrow[0, \infty)$ such that $\lim _{\epsilon \backslash \searrow_{0}} K(\epsilon)=0$, and $\forall \epsilon>0, \exists$ $\delta>0$ such that ${ }^{4} \forall x_{0} \in B_{\delta}\left(\Delta\left(x^{*}\right)\right), \operatorname{dist}\left(\phi_{T_{I}}^{\epsilon}\left(x_{0}\right), \mathcal{Z}\right) \leq K(\epsilon) \operatorname{dist}\left(x_{0}, \mathcal{Z}\right)$.

Then the restriction dynamics $\left.\Sigma^{c l, \epsilon}\right|_{\mathcal{Z}}=\left(\mathcal{Z}, \mathcal{S} \cap \mathcal{Z},\left.\Delta\right|_{\mathcal{S} \cap \mathcal{Z}},\left.f\right|_{\mathcal{Z}}\right)$ is independent of $\epsilon$. In addition, there exists $\bar{\epsilon}>0$ such that for $0<\epsilon<\bar{\epsilon}$, the following are equivalent:

i) $x^{*}$ is an exponentially stable fixed point of $\rho$, and

ii) $x^{*}$ is an exponentially stable fixed point of $P^{\epsilon}$, where $P^{\epsilon}=\phi_{T_{I}}^{\epsilon} \circ \Delta$ and $\rho=\left.P^{\epsilon}\right|_{\mathcal{Z}}$.

${ }^{3} \Gamma_{1}(x)$ and $\Gamma_{2}(x)$ refer to the first $k-1$ and last $n-k$ coordinates of $\Gamma(x)$, and $\Psi_{1}(x)$ and $\Psi_{2}(x)$ refer to the first $k$ and last $n-k$ coordinates of $\Psi(x)$, respectively.

${ }^{4}$ Throughout the paper, the notation $B_{r}(x)$ refers to an open ball of radius $r$ about the point $x$. 
The proof of the theorem is given in the appendix and shows that H2.3) is sufficient for achieving $\lim _{\epsilon \backslash 0} S_{\phi_{T_{I}}}^{\epsilon}=0$. In other words, for $\epsilon>0$ sufficiently small, an exponentially stable

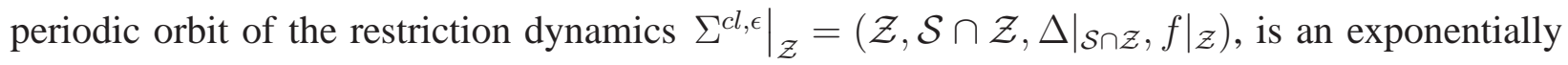
stable periodic orbit of the full model $\Sigma^{c l, \epsilon}=\left(\mathcal{X}, \mathcal{S}, \Delta, f^{\epsilon}\right)$. The next result shows how to construct a closed-loop system meeting the hypotheses of Corollary 2.

Definition 3: (from [21]) An output $h(x)$ has uniform vector relative degree $k$ at a point $x$ if

i) for $0 \leq n \leq k-2, L_{g} L_{f}^{n} h(x)=0$; and

ii) the matrix $L_{g} L_{f}^{k-1} h(x)$ is invertible.

Corollary 4: Assume that a control system with impulse effects $\Sigma^{o l}=(\mathcal{X}, \mathcal{S}, \emptyset, \mathcal{U}, \Delta, f, g)$, has a smooth output $y=h(x), h: \mathcal{X} \rightarrow \mathbb{R}^{m}$, with the properties that

H4.1) $h(x)$ has uniform vector relative degree $k$;

H4.2) there exists a point $x$ such that $h(x)=0, L_{f} h(x)=0, \ldots, L_{f}^{k-1} h(x)=0$; and

H4.3) the distribution $\operatorname{span}\left\{g_{1}(x), \cdots, g_{m}(x)\right\}$ is involutive.

Then the set $\mathcal{Z}=\left\{x \in \mathcal{X} \mid h(x)=0, L_{f} h(x)=0, \cdots, L_{f}^{k-1} h(x)=0\right\}$ is a smooth embedded submanifold. Moreover, for any $\epsilon>0$ and any scalar constants $K_{0}, \ldots, K_{k-1}$ where $s^{k}+$ $K_{k-1} s^{k-1}+\cdots K_{0}$ is Hurwitz, the feedback

$$
u^{\epsilon}(x)=-\left(L_{g} L_{f}^{k-1} h(x)\right)^{-1}\left(L_{f}^{k} h(x)+\sum_{i=0}^{k-1} \frac{1}{\epsilon^{k-i}} K_{i} L_{f}^{i} h(x)\right),
$$

applied to $\Sigma^{o l}$ renders $\mathcal{Z}$ forward invariant in the family of closed-loop systems $\Sigma^{c l, \epsilon}=\left(\mathcal{X}, \mathcal{S}, \Delta, f^{c l, \epsilon}\right)$ for $f^{c l, \epsilon}(x)=f(x)+g(x) u^{\epsilon}(x)$. In addition, the family of systems $\Sigma^{c l, \epsilon}$ and the manifold $\mathcal{Z}$ satisfy conditions H2.1) to H2.3) of Corollary 2.

The proof is given in the appendix. Theorem 1 and Corollaries 2 and 4 provide precise guidelines for designing a closed-loop system where the stability of a periodic orbit can be determined on the basis of a restriction dynamics. This is of practical importance because the restriction dynamics necessarily has lower dimension than the full model, and usually the dimension is significantly lower. The main obstacle to applying these results is achieving impact invariance of the forward invariant manifold $\mathcal{Z}$, which is addressed in the following section. 


\section{Achieving Impact Invariance Through Hybrid Extension}

\section{A. Definition and Properties of Hybrid Extensions}

Let $\Sigma^{o l}=(\mathcal{X}, \mathcal{S}, \emptyset, \mathcal{U}, \Delta, f, g)$ be a control system with impulse effects ${ }^{5}$ and let $\mathcal{A}$ be an open subset of $\mathbb{R}^{q}, q \geq 1$. Then, the system

$$
\Sigma_{e}^{o l}:\left\{\begin{array}{rlrl}
(\dot{x}, \dot{\alpha}) & =(f(x)+g(x) u, 0) & \left(x^{-}, \alpha^{-}\right) \notin \mathcal{S} \times \mathcal{A} \\
\left(x^{+}, \alpha^{+}\right)=\left(\Delta\left(x^{-}\right), v\right) & \left(x^{-}, \alpha^{-}\right) \in \mathcal{S} \times \mathcal{A},
\end{array}\right.
$$

is called a parameterized extension of $\Sigma^{o l}$ and is denoted as $\Sigma_{e}^{o l}=\left(\mathcal{X}_{e}, \mathcal{S}_{e}, \mathcal{A}, \mathcal{U}, \Delta_{e}, f_{e}, g_{e}\right)$ (with elements of the 7-tuple $\Sigma_{e}^{o l}$ defined in the obvious way).

When a parameter update law $v$ is chosen to be independent of the parameter $\alpha$, that is, $v: \mathcal{S} \rightarrow \mathcal{A}$, the resultant system $\Sigma_{e}^{o l}=\left(\mathcal{X}_{e}, \mathcal{S}_{e}, \emptyset, \mathcal{U}, \Delta_{e}^{c l}, f_{e}, g_{e}\right)$, with $\Delta_{e}^{c l}\left(x_{e}\right)=(\Delta(x), v(x))$ and $x_{e}=(x, \alpha)$, is called an open-loop deadbeat hybrid extension. A closed-loop deadbeat hybrid extension is denoted $\Sigma_{e}^{c l}=\left(\mathcal{X}_{e}, \mathcal{S}_{e}, \Delta_{e}^{c l}, f_{e}^{c l}\right)$ where $f_{e}^{c l}\left(x_{e}\right)=f_{e}\left(x_{e}\right)+g_{e}\left(x_{e}\right) u\left(x_{e}\right)$ for some continuous-time state feedback $u: \mathcal{X}_{e} \rightarrow \mathcal{U}$.

Remark 5: Suppose that $\Sigma^{o l}=(\mathcal{X}, \mathcal{S}, \emptyset, \mathcal{U}, \Delta, f, g)$ has a periodic orbit $\mathcal{O}$. Define the parameter vector $\alpha^{*}=v\left(x^{*}\right)$ for $x^{*}=\overline{\mathcal{O}} \cap \mathcal{S}$. Then the set $\mathcal{O}_{e}=\mathcal{O} \times \alpha^{*}$ is a periodic orbit of the open-loop deadbeat hybrid extension $\Sigma_{e}^{o l}$, and $\mathcal{O}_{e}$ will be called the trivial lift of $\mathcal{O}$ into $\Sigma_{e}^{o l}$.

Remark 6: Consider a closed-loop deadbeat hybrid extension $\Sigma_{e}^{c l}=\left(\mathcal{X}_{e}, \mathcal{S}_{e}, \Delta_{e}^{c l}, f_{e}^{c l}\right)$ and let $P_{e}$ be its Poincaré map. If $\Sigma_{e}^{c l}$ contains a hybrid invariant manifold $\mathcal{Z}_{e}$ and there exists a set $\mathcal{Z} \subset \mathcal{Z}_{e}$ such that $\mathcal{S}_{e} \cap \mathcal{Z}_{e}=(\mathcal{S} \cap \mathcal{Z}) \times \mathcal{A}$, then the Poincaré return map for the restriction dynamics $\left.\Sigma_{e}^{c l}\right|_{\mathcal{Z}_{e}}$ has the property that $\left.P_{e}\right|_{\mathcal{Z}_{e}}:(\mathcal{S} \cap \mathcal{Z}) \times \mathcal{A} \rightarrow(\mathcal{S} \cap \mathcal{Z}) \times \mathcal{A}$, by

$$
\left.P_{e}\right|_{\mathcal{Z}_{e}}(z, \alpha)=\left(\rho_{e}(z), v(z)\right)
$$

where $v$ is the parameter update law of the deadbeat hybrid extension and $\rho_{e}: \mathcal{S} \cap \mathcal{Z} \rightarrow \mathcal{S} \cap \mathcal{Z}$.

\section{B. Constructing Hybrid Invariant Manifolds}

The following theorem, with constructive proof in the appendix, addresses the property of impact invariance.

${ }^{5}$ Note that $\mathcal{V}=\emptyset$, indicating an absence of control authority over the impact map. 
Theorem 7: Consider a smooth control system with impulse effects ${ }^{6} \Sigma^{o l}=(\mathcal{X}, \mathcal{S}, \emptyset, \mathcal{U}, \Delta, f, g)$, with $\mathcal{U} \subset \mathbb{R}^{m}$. Assume there exists a periodic orbit $\mathcal{O}$ that is transversal to $\mathcal{S}$ and that in addition H7.1) there exists a smooth output $h: \mathcal{X} \rightarrow \mathbb{R}^{m}$ such that $h$ vanishes on the orbit and has uniform vector relative degree $k$ in an open neighborhood of the orbit;

H7.2) the distribution $\operatorname{span}\left\{g_{1}(x), \cdots, g_{m}(x)\right\}$ is involutive; and

H7.3) there exists a smooth real-valued function $\tau(x)$ that is strictly monotonically increasing ${ }^{7}$ on $\mathcal{O}, L_{g} \tau(x)=\cdots=L_{g} L_{f}^{k-2} \tau(x)=0$, and for $x^{*}=\overline{\mathcal{O}} \cap \mathcal{S}, \tau\left(x^{*}\right)=1$ and $\tau\left(\Delta\left(x^{*}\right)\right)=0$.

Then, starting from the original system $\Sigma^{o l}$ and output function $y=h(x)$, one can construct an open-loop deadbeat hybrid extension $\Sigma_{e}^{o l}=\left(\mathcal{X}_{e}, \mathcal{S}_{e}, \emptyset, \mathcal{U}, \Delta_{e}^{c l}, f_{e}, g_{e}\right)$ and a new output function $y_{e}=h_{e}\left(x_{e}\right)$ such that all of the conditions of Corollary 4 are satisfied for $\Sigma_{e}^{o l}$ and $h_{e}$. Moreover, the manifold $\mathcal{Z}_{e}$ defined as

$$
\mathcal{Z}_{e}=\left\{x_{e} \in \mathcal{X}_{e} \mid h_{e}\left(x_{e}\right)=0, L_{f_{e}} h_{e}\left(x_{e}\right)=0, \cdots, L_{f_{e}}^{k-1} h_{e}\left(x_{e}\right)=0\right\},
$$

is impact invariant w.r.t. $\Sigma_{e}^{o l}$ and contains $\mathcal{O}_{e}$, the trivial lift of $\mathcal{O}$ into $\Sigma_{e}^{o l}$.

Remark 8: The derivation of the parameter update law of the open-loop deadbeat hybrid extension of Theorem 7 allows the introduction of a parameter $\lambda \in \mathbb{R}$. Dependence of the parameter update law on $\lambda$ will be emphasized using the notation $v^{\lambda}\left(x^{-}\right)$. When $\lambda$ is chosen to equal zero, $\alpha^{+}=v^{\lambda}\left(x^{-}\right)$is such that for any $\left(x^{-}, \alpha^{-}\right) \in \mathcal{S}_{e}$,

$$
h_{e}\left(x^{+}, \alpha^{+}\right)=0, L_{f_{e}} h_{e}\left(x^{+}, \alpha^{+}\right)=0, \ldots, L_{f_{e}}^{k-1} h_{e}\left(x^{+}, \alpha^{+}\right)=0 .
$$

where $x^{+}=\Delta\left(x^{-}\right)$. Furthermore, $v^{\lambda}$ has the following two properties:

i) for any fixed $x_{e}^{-}=\left(x^{-}, \alpha^{-}\right) \in \mathcal{S}_{e}$, the value of $v^{\lambda}\left(x^{-}\right)$is continuous in $\lambda$; and

ii) for any fixed $x_{e}^{-}=\left(x^{-}, \alpha^{-}\right) \in \mathcal{S}_{e} \cap \mathcal{Z}_{e}$, the value of $v^{\lambda}\left(x^{-}\right)$is independent of $\lambda$.

Remark 9: An $\epsilon$ and $\lambda$ dependant closed-loop deadbeat hybrid extension can be created by applying Corollary 4 to the open-loop deadbeat hybrid extension constructed in Theorem 7.

\footnotetext{
${ }^{6}$ Once again note that $\mathcal{V}=\emptyset$, indicating an absence of control authority over the impact map.

${ }^{7}$ In this context, a real-valued function $\tau(x)$ is strictly monotonically increasing if $L_{f} \tau(x)>0$ for every point $x$ in $\mathcal{O}$.
} 
Applying Corollary 4 and Theorem 1, it follows that for any $\lambda \in \mathbb{R}$ and for $z_{e}^{*}=\left(z^{*}, \alpha^{*}\right)$,

$$
\mathcal{D} \hat{P}_{e}^{\epsilon, \lambda}\left(z_{e}^{*}, \eta_{e}^{*}\right)=\left[\begin{array}{c|c}
\mathcal{D}\left(P_{e} \mid z_{e}\right)\left(z_{e}^{*}\right) & \star \\
\hline 0 & S_{\phi_{T_{I}}, e}^{\epsilon}\left(\bar{z}_{e}^{*}, \bar{\eta}_{e}^{*}\right) S_{\Delta, e}^{\lambda}\left(z_{e}^{*}, \eta_{e}^{*}\right)
\end{array}\right],
$$

with the subscript " $e$ " indicating reference to the closed-loop deadbeat hybrid extension. Because $\mathcal{Z}_{e}$ meets the conditions of Remark 6,

$$
\mathcal{D} \hat{P}_{e}^{\epsilon, \lambda}\left(z_{e}^{*}, \eta_{e}^{*}\right)=\left[\begin{array}{c|c|c}
\mathcal{D} \rho_{e}\left(z^{*}\right) & 0 & \star \\
\hline 0 & 0 & \star \\
\hline 0 & 0 & S_{\phi_{T}, e}^{\epsilon}\left(\bar{z}_{e}^{*}, \bar{\eta}_{e}^{*}\right) S_{\Delta, e}^{\lambda}\left(z_{e}^{*}, \eta_{e}^{*}\right)
\end{array}\right] .
$$

As shown in the proof of Corollary 2, $\lim _{\epsilon} \backslash 0 S_{\phi_{T_{I}}}^{\epsilon}\left(\bar{z}_{e}^{*}, \bar{\eta}_{e}^{*}\right)=0$, and by (12) and property i) of $v, \lim _{\lambda \rightarrow 0} S_{\Delta, e}^{\lambda}\left(z_{e}^{*}, \eta_{e}^{*}\right)=0$. Because the vector field $\left.f_{e}^{c l, \epsilon}\right|_{\mathcal{Z}_{e}}$ is independent of $\epsilon$ and $\left.v^{\lambda}\right|_{\mathcal{S}_{e} \cap \mathcal{Z}_{e}}$ is independent of $\lambda$ (by property ii of $v$ ), it follows that $\mathcal{D} \rho_{e}\left(z^{*}\right)$ is independent of both $\epsilon$ and $\lambda$. Thus, for appropriate choices of $\lambda$ and $\epsilon, \max \left|\operatorname{eig}\left(\mathcal{D} P_{e}^{\epsilon, \lambda}\left(x_{e}^{*}\right)\right)\right|=\max \left|\operatorname{eig}\left(\mathcal{D} \rho_{e}\left(z^{*}\right)\right)\right|$, showing how the creation of a hybrid zero dynamics leads to a low-dimensional test for stabilizability of an orbit.

\section{Application to Planar Bipedal Walkers with Compliant Actuation}

To give an application of theory, this section develops a class of models for planar robots with series compliant actuation (see Fig. 2), derives properties of these models as they relate to planar robots without series compliance, and provides a simulation study of a sample gait stabilized by the continuous-time feedback of Corollary 4 and the parameter updates of Theorem 7. As shown in Fig. 2, a series compliant actuator is one in which a compliant element has been deliberately inserted between an actuated joint and its corresponding motor in order to increase the overall energy efficiency or to more accurately control the torques applied at the joint. The specific model used in simulation is based on a prototype robot that is currently under construction in a collaborative effort between the University of Michigan and Carnegie Mellon University. The purpose of the robot, named BiMASC, is to study the effects of series compliant actuation on achieving efficient, stable, planar walking and running motions.

Including springs in a legged robot is a well-motivated choice [2], [1]. Introducing tuned springs into an otherwise rigid mechanism can significantly improve energy efficiency in two ways: within the strides of walking and running, springs can store and release some of the energy 
that would otherwise be lost as actuators do negative work, and at foot touchdown events, springs isolate reflected motor inertias from the energy-dissipating effects of rigid collisions.

However, obtaining the energetic benefits of compliance is not without cost: delivering torque through compliant elements poses several challenges for control design. There is an obvious increase in the degrees of freedom of the robot, and hence, the degree of underactuation. This is a widely recognized issue in robotics; see [31], [30], [4] and references therein. An additional challenge particular to legged robots arises from the impulsive effects occurring when the swing leg impacts the ground. Because of the higher degree of underactuation, previous results such as [33] are not applicable to bipeds with series compliant actuation, further motivating the theory of Sections III and IV.

\section{A. Hybrid Models}

As in [34, Sec. II], consider a bipedal robot consisting of $N$ links connected in a planar tree structure to form two identical legs with knees, but without feet, with the legs connected at a common point called the hips, and possibly other limbs (such as a torso, etc.). All links have mass, are rigid, and are connected in revolute joints (see Fig. 2). It is assumed that no actuation is applied between the stance leg and the ground, while all other joints are independently actuated, and hence there are $(N-1)$ controls. As shown in [12], [13], [14], addressing the control of robots without actuation between the stance leg and ground is an important step in achieving anthropomorphic walking motions in robots with non-trivial feet and actuated ankles. Further details on the model are given in [34, Sec. II], along with assumptions on the walking gait (instantaneous double support phase, no slipping nor rebound at impact, motion from left to right, symmetric gait). A rigid impact is used to model the contact of the swing leg with the ground. To study the effects of introducing springs, a rigid model (without springs) is first derived, followed by a compliant model having series compliant actuation at each body coordinate.

The configuration coordinates of the robot in single support (also commonly called the stance phase) are denoted by $q=\left(q_{1}, \cdots, q_{N}\right)$. The method of Lagrange leads to the mechanical model for the rigid walker,

$$
D(q) \ddot{q}+C(q, \dot{q}) \dot{q}+G(q)=B u
$$

where $D(q)$ is the inertia matrix, $C(q, \dot{q})$ contains Coriolis and centrifugal terms, $G(q)$ is the gravity vector, and $B$ is an $N \times(N-1)$ constant matrix with rank $(N-1)$. Letting $x=(q, \dot{q})$, 

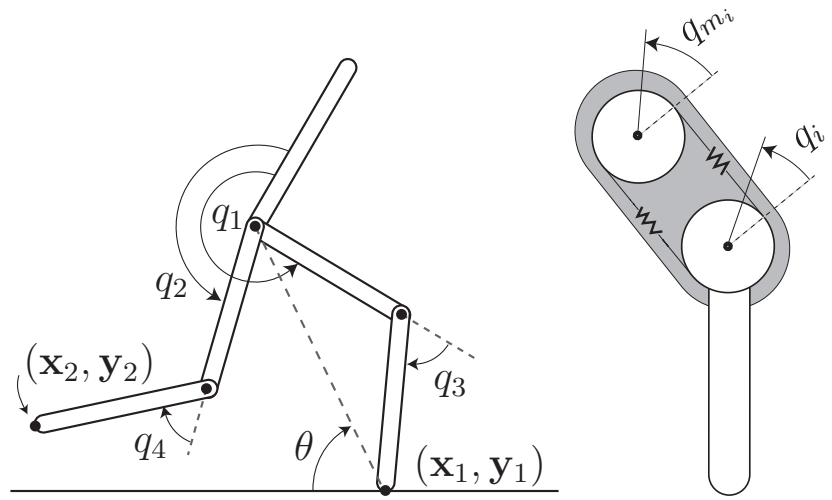

Fig. 2. (Left) A coordinate diagram of an example of the class of $N$-link biped robot models considered. (Right) A schematic of a rotational joint with series compliant actuation.

and defining $f$ and $g$ in the obvious manner, the mechanical model is expressed in state variable form as

$$
\dot{x}=f(x)+g(x) u
$$

When the swing leg contacts the ground, an inelastic impact is assumed, giving rise to a jump in the velocity coordinates, which is computed as in [20], [16]. So that the same mechanical model can be used independent of which leg is the stance leg, the coordinates must also be relabeled at impact, giving rise to a jump in the configuration variables as well; see [16], [34].

The hybrid model of the robot (stance phase Lagrangian dynamics plus impact map) is constructed by specifying a state manifold, $\mathcal{X} \subset \mathbb{R}^{2 N}$, corresponding to physically reasonable joint configurations and velocities, the impact or switching surface

$$
\mathcal{S}=\left\{(q, \dot{q}) \in \mathcal{X} \mid \mathbf{y}_{2}(q)-\mathbf{y}_{1}(q)=0, \mathbf{x}_{2}(q)-\mathbf{x}_{1}(q)>0\right\}
$$

the set of points where the swing leg height is zero and is horizontally in front of the stance leg, and the impact map $\Delta: \mathcal{S} \rightarrow \mathcal{X}$. The corresponding system with impulse effects for the rigid model is $\Sigma_{r}^{o l}=\left(\mathcal{X}, \mathcal{S}, \emptyset, \mathbb{R}^{N-1}, \Delta, f, g\right)$.

Assume now that the vector of torques applied to the robot model (13) is generated through a compliant model to form the $(2 N-1)$ DOF Lagrangian system

$$
\begin{aligned}
D(q) \ddot{q}+C(q, \dot{q}) \dot{q}+G(q)-B K\left(q_{m}-q_{a}\right) & =0 \\
J \ddot{q}_{m}+K\left(q_{m}-q_{a}\right) & =u_{c} .
\end{aligned}
$$


where $q_{m}$ is a vector of $(N-1)$ motor angles, $q_{a}$ is a vector of the relative angles corresponding to the $(N-1)$ actuated joints of the robot, $u_{c}$ is the vector of $(N-1)$ motor torques, $K$ is a diagonal matrix of (positive) spring constants and $J$ is a diagonal matrix of (positive) rotor inertias. Letting $\mathcal{X}_{c}=\mathcal{X} \times \mathbb{R}^{2(N-1)}$ and $x_{c}=\left(x, q_{m}, \dot{q}_{m}\right)$, this is easily expressed as

$$
\dot{x}_{c}=f_{c}\left(x_{c}\right)+g_{c}\left(x_{c}\right) u_{c}
$$

Because the impact condition depends only on the position of the swing foot (which is independent of the motor variables), $\mathcal{S}_{c}=\mathcal{S} \times \mathbb{R}^{2(N-1)}$. Following [20], [16], the impact map $\Delta_{c}: \mathcal{S}_{c} \rightarrow \mathcal{X}_{c}$ has the form $\Delta_{c}\left(x_{c}^{-}\right)=\left(\Delta\left(x^{-}\right), R q_{m}^{-}, R \dot{q}_{m}^{-}\right)$, where $R$ is a joint relabeling matrix. Up to joint relabeling, the impact map $\Delta_{c}$ imposes continuity ${ }^{8}$ in the motor positions and velocities across the impact. The corresponding model with impulse effects for the compliant model is written as $\Sigma_{c}^{o l}=\left(\mathcal{X}_{c}, \mathcal{S}_{c}, \emptyset, \mathbb{R}^{N-1}, \Delta_{c}, f_{c}, g_{c}\right)$.

\section{B. Model Properties}

Some properties of the mechanical models (13) and (15) are now summarized. These properties provide information on the stance phase zero dynamics of (14) and (16). In the following, we choose configuration coordinates for (13) as $q=\left(q_{a}, \theta\right)$, where $\theta$ references a position on the robot to the world frame (see Fig 2).

Proposition 10: Let $\sigma$ be the angular momentum of the biped about the contact point of the support leg with the ground. In the coordinates $q=\left(q_{a}, \theta\right)$,

a) the inertia matrix $D$ of (13) is independent of $\theta$;

b) (14) is globally feedback equivalent to

$$
\begin{aligned}
\dot{\sigma} & =-\frac{\partial V}{\partial \theta}(q) \\
\dot{\theta} & =\frac{\sigma}{d_{N N}\left(q_{a}\right)}+R\left(q_{a}\right) \dot{q}_{a} \\
\ddot{q}_{a} & =w,
\end{aligned}
$$

where $V$ is the potential energy of the robot model (13),

$$
R\left(q_{a}\right)=-\left[\frac{d_{N, 1}\left(q_{a}\right)}{d_{N, N}\left(q_{a}\right)}, \cdots, \frac{d_{N, N-1}\left(q_{a}\right)}{d_{N, N}\left(q_{a}\right)}\right]
$$

\footnotetext{
${ }^{8}$ In other words, the springs isolate the motors' rotor inertias from the impact dynamics. Since in practice, the rotor inertias are reflected through a gear ratio on the order of $10: 1$ or higher, removing the rotor inertias from the impact dynamics can result in considerably less energy loss at impacts.
} 
and $d_{i, j}$ are the elements of $D$; and

c) (16) is globally feedback equivalent to

$$
\begin{aligned}
\dot{\sigma} & =-\frac{\partial V}{\partial \theta}(q) \\
\dot{\theta} & =\frac{\sigma}{d_{N N}\left(q_{a}\right)}+R\left(q_{a}\right) \dot{q}_{a} \\
q_{a}^{(4)} & =w .
\end{aligned}
$$

The proof and the required feedback are given in [17], and are based on [32], [28].

Proposition 11: Include the same smooth output function $y=h(q)$ in the rigid model $\Sigma_{r}^{o l}$ and the compliant model $\Sigma_{c}^{o l}$. Then the following hold,

a) $h$ has uniform vector relative degree 2 for (14) if, and only if, it has uniform vector relative degree 4 for (16);

b) the decoupling matrices depend only on $q$ and they are equal, that is $L_{g} L_{f} h=L_{g_{c}} L_{f_{c}}^{3} h$;

c) for $h(q)=q_{a}-h_{d}(\theta), \operatorname{det}\left(L_{g} L_{f} h\right)(q)=1-R\left(q_{a}\right) \frac{\partial h_{d}(\theta)}{\partial \theta}$;

d) if $h(q)=q_{a}-h_{d}(\theta)$ and has uniform vector relative degree 2 for (14), then the zero dynamics manifolds and restriction dynamics of (14) and (16) are diffeomorphic. Moreover, the zero dynamics manifold of (14) is

$$
\mathcal{Z}=\left\{(q, \dot{q}) \in \mathcal{X} \mid q_{a}=h_{d}(\theta), \dot{q}_{a}=\frac{\partial h_{d}(\theta)}{\partial \theta} \dot{\theta}\right\}
$$

and in the coordinates $(\theta, \sigma)$, the restriction dynamics is

$$
\begin{aligned}
\dot{\sigma} & =\left.\frac{-\partial V}{\partial \theta}\right|_{q_{a}=h_{d}(\theta)} \\
\dot{\theta} & =\frac{\sigma}{\tilde{d}_{N, N}(\theta)}\left(1-\tilde{R}(\theta) \frac{\partial h_{d}(\theta)}{\partial \theta}\right)^{-1},
\end{aligned}
$$

where, $\tilde{d}_{N, N}(\theta)=\left.d_{N, N}\right|_{q_{a}=h_{d}(\theta)}$ and $\tilde{R}(\theta)=\left.R\right|_{q_{a}=h_{d}(\theta)}$.

Proof: a) and b) are immediate from Proposition 10. The calculation for c) is a straightforward application of the Sherman-Morrison-Woodbury formula [6] and is left to the reader. The first part of d) follows from basic results in [21] and equations (17), (18), and (19) follow from [34].

\section{Designing a Gait, Outputs, and a Monotonic Function}

Various computational methods have been proposed to design gaits for models similar to those used here. In order to implement the control methods of this paper on a robot model 
with compliance, $\Sigma_{c}^{o l}$, we need not only a reference gait (periodic orbit), but also (from H7.1) a smooth output $h: \mathcal{X}_{c} \rightarrow \mathbb{R}^{m}$ that vanishes on the orbit and has uniform vector relative degree $k$, and (from H7.3) a smooth real-valued function $\tau\left(x_{c}\right)$ that is strictly monotonic on the orbit and satisfies $L_{g_{c}} \tau\left(x_{c}\right)=\cdots=L_{g_{c}} L_{f_{c}}^{k-2} \tau\left(x_{c}\right)=0$. The example gait of Fig. 3 was found by first choosing a family of output functions ${ }^{9}$ parameterized by a vector $\beta$, namely

$$
y=h(q, \beta)=q_{a}-h_{d}(\theta, \beta),
$$

where $h_{d}$ is, in the following example, a $4 \times 1$ vector of $7^{\text {th }}$ degree Bézier polynomials. Gradient optimization was used to find a $4 \times 8$ matrix $^{10}$ of polynomial coefficients $\beta^{*}$ and an initial condition $x_{0} \in \mathcal{X}_{c}$ such that

i) $x_{0}$ lies in a periodic orbit $\mathcal{O}$ of the system $\Sigma_{c}^{o l}$ under the state feedback controller

$$
u_{c}\left(x_{c}\right)=-\left(L_{g_{c}} L_{f_{c}}^{k-1} h\left(x_{c}, \beta^{*}\right)\right)^{-1}\left(L_{f_{c}}^{k} h\left(x_{c}, \beta^{*}\right)\right)
$$

ii) the outputs $y=q_{a}-h_{d}\left(\theta, \beta^{*}\right)$ vanish on the orbit $\mathcal{O}$ and have uniform vector relative degree $k$ in an open neighborhood of $\mathcal{O}$; and

iii) the coordinate $\theta$ is strictly monotonic on the orbit.

In the presence of the geometric constraints of ii) and iii), numerical integration of the stance phase dynamics can be efficiently carried out on a lower-dimensional system, which is the stance phase zero dynamics; see [35].

When numerical optimization is successful, the resulting gait and output function satisfy the hypotheses of Corollary 4. As a result, there exists a feedback such that the closed-loop system satisfies the conditions of Corollary 2 and all the hypotheses of Theorem 1 except H1.1). This last condition is the most difficult to satisfy and, in general, requires parameter updates such as those developed in the proof of Theorem 7 and discussed in the following subsection.

\section{Achieving Hybrid Invariance}

By Proposition 10, the compliant model is a dynamic extension of the rigid model, and hence by [21], the problem of designing controllers to zero an output of the form (20) is, from a

\footnotetext{
${ }^{9}$ In an alternative method using techniques of [37], the orbit could be designed first, independently of the outputs. Compatible outputs $y=h\left(x_{c}\right)$ and a monotonic $\tau\left(x_{c}\right)$ are chosen in a second step.

${ }^{10}$ Each $7^{\text {th }}$ degree polynomial has 8 independent coefficients.
} 
theoretical perspective, no more difficult for the compliant model than for the rigid model. In particular, parts (a) and (b) of Proposition 10 show that if an output function $h(q)$ for the rigid model, $\Sigma_{r}^{o l}$, satisfies H4.1), H4.2), and H4.3) of Corollary 4, then the same output function when used with the compliant model, $\Sigma_{c}^{o l}$, will also satisfy H4.1), H4.2), and H4.3) of Corollary 4. Creating a forward invariant manifold is straightforward in each case.

However, the stability test of Theorem 1 requires the additional property of impact invariance of $\mathcal{Z}$-which is significantly harder to achieve in the compliant model than in the rigid one, due to the higher relative degree of the output. For outputs having the special form of (20), when used with planar biped models with one degree of underactuation, a rigid impact map, and no compliance, it can be shown that any forward invariant manifold $\mathcal{Z}$ containing a periodic orbit $\mathcal{O}$ is automatically impact invariant [36, Thm. 6.2]. Such a result does not hold for the compliant model, and in fact, we (the authors) have been unable to find even a single example of an output of the form (20) giving rise to an impact invariant manifold in the model with compliance, without the use of parameter updates. We conjecture that outputs of this form never give rise to an impact invariant manifold when actuation is provided through springs.

Theorem 7 shows how impact invariance can be recovered through the introduction of a discrete component to the overall control law, where a parameterized output is introduced and the parameters are updated stride to stride. This procedure gives rise to a manifold that is hybrid invariant, allowing the stability test of Theorem 1 to be carried out on an orbit that is a trivial lift of the original. See Remark 5.

To contrast this result with previous work, in [25] we found a parameterized output function, continuous-phase controller, and impact update law to render the resulting manifold hybrid invariant in a closed-loop deadbeat hybrid extension of a five-link robot model with compliance. In that work, the parameterized output function and appropriate parameter update law were crafted by hand and heavily exploited the fact that the underlying rigid model had only one degree of underactuation. Based strongly on spline-like transition functions, the constructive outputs proposed in this paper are significantly different from those used in [25] and the design procedure does not constrain the number of degrees of underactuation. 


\section{E. Simulation on a five-link biped}

A numerical example is provided here to illustrate the application of the theorems of this paper to the task of stabilizing walking in a five-link planar biped with series compliant actuation. A gait was designed following the method of [35] to achieve a forward progression rate of $0.8 \mathrm{~m} / \mathrm{s}$ and to minimize an approximation of motor electrical energy consumed per distance traveled, subject to constraints on max torque, ground friction, joint limits, etc. Parameters of the model are available in Table I and are estimates of the physical parameters of BiMASC. In the model, all links have uniform mass distributions except the femurs, whose center of mass is $0.15 \mathrm{~m}$ from the hip joint. Figure 3 gives a stick animation of the sample gait. Values of $\theta$ below each frame show that $\theta$ is monotonically increasing within a stride. The percentage value indicates the amount of total step time elapsed, which has a nonlinear relationship with $\theta$. Rotor angles for the sample motion are shown in Fig. 4. As required by the impact model, values of rotor position and velocity are constant across the impact event, up to joint relabeling.

The controller for stabilizing this gait was derived by choosing $\lambda=1$ and constructing an openloop deadbeat hybrid extension, $\Sigma_{c, e}^{o l, \lambda}=\left(\mathcal{X}_{c, e}, \mathcal{S}_{c, e}, \emptyset, \mathbb{R}^{4}, \Delta_{c, e}^{c l, \lambda}, f_{c, e}, g_{c, e}\right)$, from the compliant system, $\Sigma_{c}^{o l}=\left(\mathcal{X}_{c}, \mathcal{S}_{c}, \emptyset, \mathbb{R}^{4}, \Delta_{c}, f_{c}, g_{c}\right)$, as described in Theorem 7. A closed-loop deadbeat hybrid extension, $\Sigma_{c, e}^{c l, \epsilon, \lambda}=\left(\mathcal{X}_{c, e}, \mathcal{S}_{c, e}, \Delta_{c, e}^{c l, \lambda}, f_{c, e}^{c l, \epsilon},\right)$, was formed by applying the feedback

$$
u_{c}^{\epsilon}\left(x_{c, e}\right)=-\left(L_{g_{c, e}} L_{f_{c, e}}^{3} h_{e}\left(x_{c, e}\right)\right)^{-1}\left(L_{f_{c, e}}^{4} h_{e}\left(x_{c, e}\right)+\sum_{i=0}^{3} \frac{1}{\epsilon^{4-i}} K_{i} L_{f_{c, e}}^{i} h_{e}\left(x_{c, e}\right)\right),
$$

for $K_{0}=1, K_{1}=4, K_{2}=6, K_{3}=4, \epsilon=0.07$ to the open-loop deadbeat hybrid extension, where $h_{e}\left(x_{e}\right)$ is an output for the open-loop deadbeat hybrid extension, constructed (as described in Theorem 7) from an output (20) for a $\beta^{*}$ chosen by optimization. The required monotonic function is $\tau\left(x_{e}\right)=\left(\theta-\theta^{+}\right) /\left(\theta^{-}-\theta^{+}\right)$, where $\theta^{+}$and $\theta^{-}$are the values of the coordinate $\theta$ just after and just before impact, respectively, as evaluated on the orbit.

Figure 5 compares eigenvalues of the transverse sensitivity matrix $S_{\phi_{T_{I}}, e}^{\epsilon}\left(\bar{z}_{e}^{*}, \bar{\eta}_{e}^{*}\right) S_{\Delta, e}^{\lambda}\left(z_{e}^{*}, \eta_{e}^{*}\right)$ of the closed-loop deadbeat hybrid extension at various values of $\epsilon$ and $\lambda$. As either $\epsilon$ or $\lambda$ is held constant and the other approaches zero, the eigenvalues of the transverse sensitivity matrix converge to zero. ${ }^{11}$ Once $\max \left|\operatorname{eig}\left(S_{\phi_{T_{I}}, e}^{\epsilon}\left(\bar{z}_{e}^{*}, \bar{\eta}_{e}^{*}\right) S_{\Delta, e}^{\lambda}\left(z_{e}^{*}, \eta_{e}^{*}\right)\right)\right|<1$, the stability of the periodic

\footnotetext{
${ }^{11}$ Theory predicts that as long as $\lambda=0$, stability of the transverse dynamics can be obtained any value of $\epsilon>0$, although the region of attraction of the controller becomes vanishingly small when $\epsilon$ is large.
} 
TABLE I

PARAMETERS OF THE FIVE LINK MODEL.

\begin{tabular}{c|c|c}
\hline Parameter & Units & Value \\
\hline \hline Length of each Link & $\mathrm{m}$ & 0.5 \\
\hline Mass of the Torso & $\mathrm{kg}$ & 27.5 \\
\hline Mass of each Femur and Tibia & $\mathrm{kg}$ & 0.5 \\
\hline CoM Inertia of the Torso & $\mathrm{kg} \cdot \mathrm{m}^{2}$ & 0.5729 \\
\hline CoM Inertia of each Tibia & $\mathrm{kg} \cdot \mathrm{m}^{2}$ & 0.0104 \\
\hline CoM Inertia of each Femur & $\mathrm{kg} \cdot \mathrm{m}^{2}$ & 0.0391 \\
\hline Reflected Inertia of Rotors & $\mathrm{kg} \cdot \mathrm{m}^{2}$ & 0.03584 \\
\hline Transmission Ratio & $(\mathrm{unitless})$ & $8: 1$ \\
\hline Spring Constant & $\mathrm{N} / \mathrm{m}$ & 550 \\
\hline
\end{tabular}
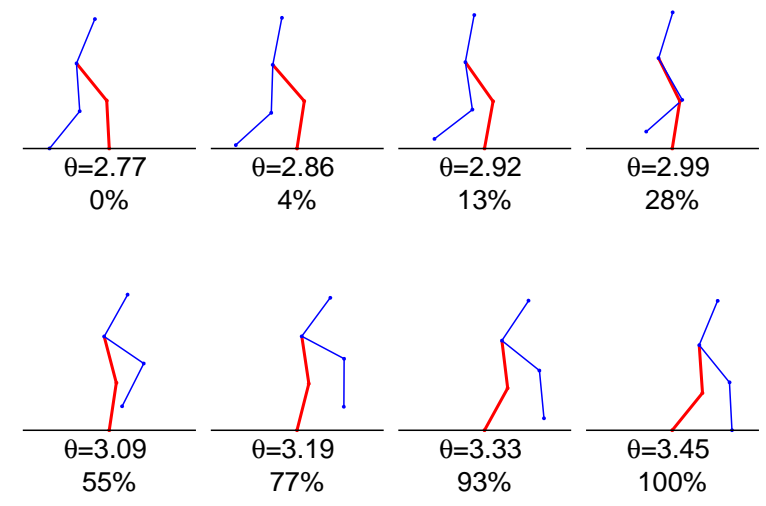

Fig. 3. Stick figure of a sample gait, walking at $0.8 \mathrm{~m} / \mathrm{s}$. Values of $\theta$ are monotonically increasing.

orbit is determined solely by the partial map $\rho_{e}$ of (11), whose eigenvalues are unaffected by either $\epsilon$ or $\lambda$. For this example, the eigenvalues of the transverse sensitivity matrix are known in closed-form as $S_{\phi_{T_{I}}, e}^{\epsilon}\left(\bar{z}_{e}^{*}, \bar{\eta}_{e}^{*}\right) S_{\Delta, e}^{\lambda}\left(z_{e}^{*}, \eta_{e}^{*}\right)=\lambda e^{A t^{*} / \epsilon}$, for $t^{*}$ equaling the period of the orbit and the constant matrix $A$ found from the polynomial coefficients $K_{0}, \ldots, K_{3}$. The one nonzero eigenvalue unaffected by either $\epsilon$ or $\lambda$ can be found as slope of $\rho_{e}$ at the fixed point; see Fig. 6.

For parameter choices of $\epsilon=0.07$ and $\lambda=1$, the magnitudes of the eigenvalues of the 

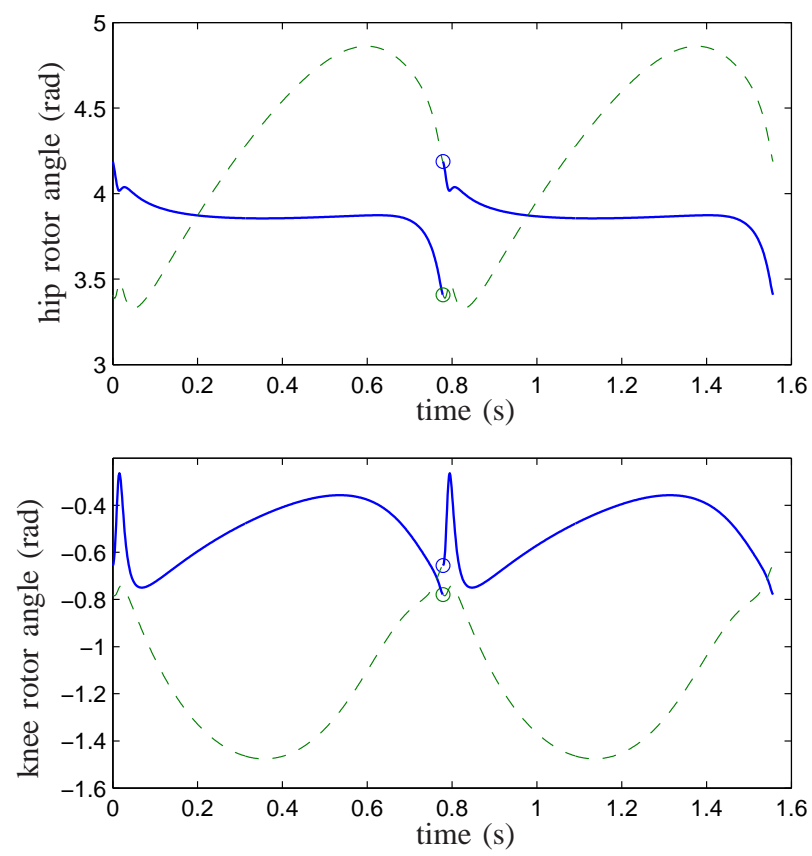

Fig. 4. Values of the motor angles $q_{m}$ along two cycles of the periodic orbit. Stance knee and hip rotors are plotted with a solid line, swing knee and hip rotors with a dashed line. Moments of impact are noted with a hollow circle. Consistent with the impact model, rotor positions and velocities are continuous across the impact.

transverse sensitivity matrix are well below zero, the eigenvalues associated with parameter updates are identically zero (see (11)), and the eigenvalue of the partial map $\rho_{e}$ is approximately equal to 0.55 -indicating that the trivial lift $\mathcal{O}_{e}$ is a stable periodic orbit in the closed-loop deadbeat hybrid extension $\Sigma_{c, e}^{c l, \epsilon, \lambda}$. A visualization of convergence is given in Fig. 7. The most important feature of this plot is that the parameters (i.e., $\alpha$ coordinates) are indeed constant within a stride, and stride-to-stride they converge to 0 . In the figure, the initial condition is marked with an asterisk and solution progresses from stride-to-stride in the direction of the arrow.

\section{CONCLUSION}

Periodic walking gaits of bipedal robots can be modeled as periodic orbits in nonlinear systems with impulse effects, with stability of an orbit determined by linearizing a Poincaré return map and checking its eigenvalues. While numerical techniques can be used to find fixed points and to estimate the Jacobian linearization of the return map about a fixed point, generally, the computations are too cumbersome for use in feedback design. One solution is to design the feedback control law so that it induces a special structure in the closed-loop system [9]. This 


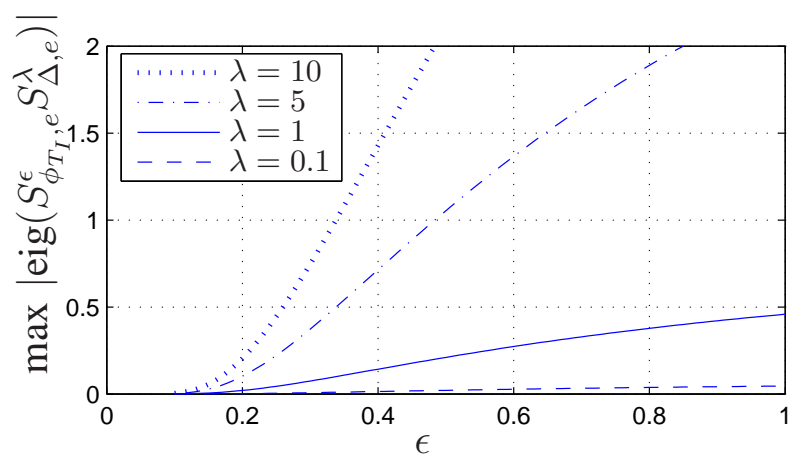

Fig. 5. Effects of the controller parameters $\epsilon$ and $\lambda$ on the eigenvalues of the product of sensitivity matrices appearing in the linearized return map. As $\lambda$ is held fixed and $\epsilon$ decreases to zero, the eigenvalues of the matrix product converge to zero. Similarly, as $\epsilon$ is held fixed and $\lambda$ approaches zero, the eigenvalues converge to zero.

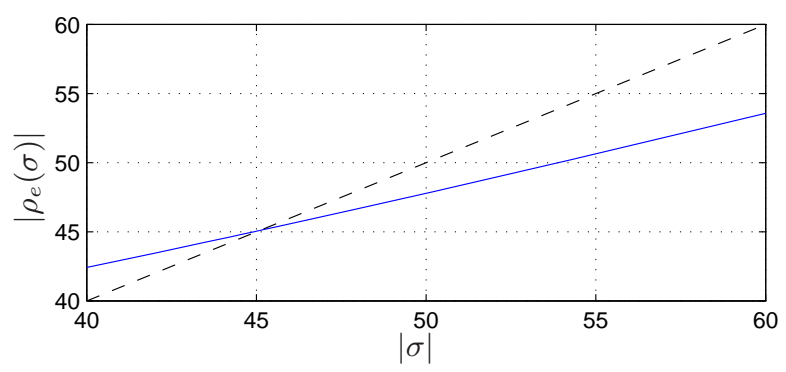

Fig. 6. The partial map $\rho_{e}$. The fixed point is located at the point where $\sigma=\rho_{e}(\sigma)$, at approximately $|\sigma|=45 \mathrm{~kg} \mathrm{~m} / \mathrm{s}$. The slope at the fixed point is approximately 0.55 , indicating that the discrete-time system $\sigma_{k+1}=\rho_{e}\left(\sigma_{k}\right)$ is locally exponentially stable. The dashed line is the identity map.

idea gave rise to the hybrid zero dynamics of nonlinear systems with impulse effects, where feedback is used to create an attractive, invariant subsystem of the hybrid model. Stability analysis of a periodic orbit can then be decomposed into the computation of the Poincare return map restricted to the invariant surface (a lower-dimensional problem) and determining the required rate of convergence of the transverse dynamics to the invariant surface [16], [34].

The current paper presents two main results that extend the analytical techniques of the hybrid zero dynamics framework. The first is a new set of hypotheses under which the stability of a periodic orbit can be determined on the basis of a restricted Poincaré map. An improvement over [34] is the elimination of an hypothesis on the existence of a particular set of coordinates. In addition, the transverse dynamics are not required to converge to the invariant surface in finite time, but instead are allowed to converge at a "sufficiently rapid" exponential rate. This allows the feedback to be smooth, whereas before it was continuous, but not Lipschitz continuous. The second main result of the paper is a constructive method for achieving hybrid invariance for a much broader class of models than was allowed by [16]. In particular, the relative degree of 


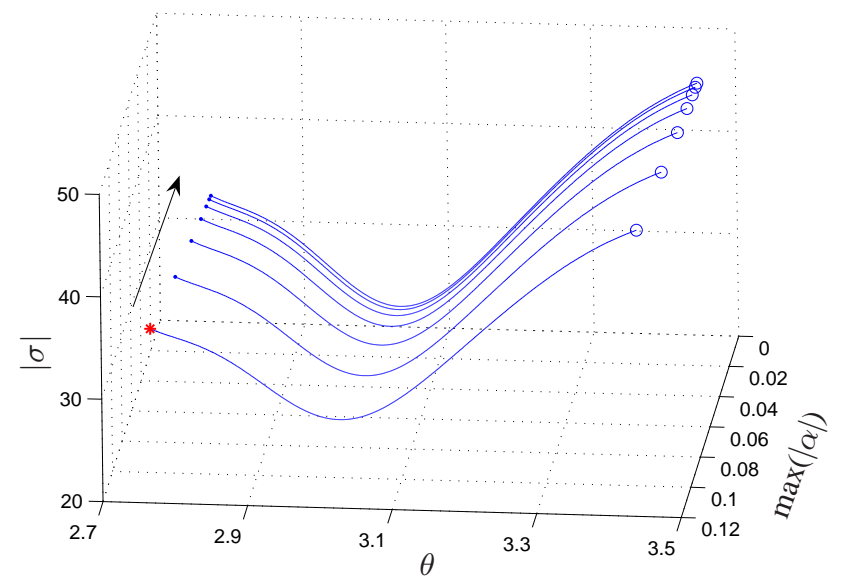

Fig. 7. Projection of a solution converging to the orbit for parameter values $\epsilon=0.07$ and $\lambda=1$. The initial condition is marked with an asterisk. Within a given stride, values of $\alpha$ are constant and converge from step to step to $\alpha=0$.

each output is no longer required to be two and special properties of the impact or reset map ${ }^{12}$ are no longer needed.

The theoretical results of the paper were demonstrated on the design of an exponentially stable, periodic walking gait for a robot with series compliant actuation. The presence of compliance allows periodic gaits that require less power for locomotion at a given speed, and hence the springs enhance autonomy. However, springs also increase the degrees of freedom of the robot, and hence the extent of its underactuation. For the example of a five-link robot with four actuated joints, the degree of underactuation becomes five. The additional degrees of underactuation pose a challenge for achieving impact invariance, that is, invariance of a surface under the impact map. Impact invariance is recovered by the use of a parameterized controller with impact updated parameters. The constructive controller design procedure of the paper was applied to a five-link robot and an exponentially stable gait was obtained.

\section{REFERENCES}

[1] M. Ahmadi and M. Buehler, "Stable control of a simulated one-legged running robot with hip and leg compliance," IEEE Transactions on Robotics and Automation, vol. 13, no. 1, pp. 96-104, February 1997.

[2] R. Alexander, “Three uses for springs in legged locomotion,” International Journal of Robotics Research, vol. 9, no. 2, pp. 53-61, 1990.

[3] A. D. Ames and R. D. Gregg, "Stably extending two-dimensional bipedal walking to three," in Proc. of the American Control Conference, 2007.

${ }^{12}$ Such as linearity in the "velocities" coordinates; see [34]. 
[4] A. Astolfi and R. Ortega, "Immersion and invariance: A new tool for stabilization and adaptive control of nonlinear systems," IEEE Transactions on Automatic Control, vol. 48, no. 4, pp. 590-606, 2003.

[5] D. Bainov and P. Simeonov, Systems with Impulse Effects : Stability, Theory and Applications. Chichester: Ellis Horwood Limited, 1989.

[6] D. S. Bernstein, Matrix mathematics : theory, facts, and formulas with application to linear systems theory. Princeton, N.J. : Princeton University Press, 2005.

[7] S. Bhat and D. Bernstein, "Finite-time stability of continuous autonomous systems," SIAM Journal of Control and Optimization, vol. 38, no. 3, pp. 751-766, 2000.

[8] W. M. Boothby, An Introduction to Differentiable Manifolds and Riemannian Geometry. New York: Academic Press, 1975.

[9] R. W. Brockett, "Pattern generation and the control of nonlinear systems," IEEE Transactions on Automatic Control, vol. 48, no. 10, pp. 1699 - 1711, October 2003.

[10] V. Chellaboina, S. P. Bhat, and W. M. Haddad, "An invariance principle for nonlinear hybrid and impulsive dynamical systems," Nonlinear Analysis, vol. 53, pp. 527-550, 2003.

[11] C. Chevallereau, E. R. Westervelt, and J. W. Grizzle, "Asymptotically stable running for a five-link, four-actuator, planar bipedal robot," International Journal of Robotics Research, vol. 24, pp. 431-464, 2005.

[12] J. H. Choi and J. W. Grizzle, "Feedback control of an underactuated planar bipedal robot with impulsive foot action," Robotica, vol. 23, no. 5, pp. 567-580, September 2005.

[13] — , "Planar bipedal walking with foot rotation," in Proc. of American Control Conference, Portland, OR, vol. 7, June 2005, pp. 4909-4916.

[14] D. Djoudi and C. Chevallereau, Fast motions in Biomechanics and Robotics. Heidelberg: Springer, 2006, ch. Stability analysis of bipedal walking with control or monitoring of the center of pressure.

[15] J. Goncalves, A. Megretski, and M. Dahleh, "Global stability of relay feedback systems," IEEE Transactions on Automatic Control, vol. 46, no. 4, pp. 550-562, April 2001.

[16] J. W. Grizzle, G. Abba, and F. Plestan, "Asymptotically stable walking for biped robots: Analysis via systems with impulse effects," IEEE Transactions on Automatic Control, vol. 46, no. 1, pp. 51-64, January 2001.

[17] J. W. Grizzle, C. Moog, and C. Chevallereau, "Nonlinear control of mechanical systems with an unactuated cyclic variable," IEEE Transactions on Automatic Control, vol. 30, no. 5, pp. 559-576, May 2005.

[18] J. Guckenheimer and P. Holmes, Nonlinear Oscillations, Dynamical Systems, and Bifurcations of Vector Fields, corrected second printing ed., ser. Applied Mathematical Sciences. New York: Springer-Verlag, 1996, vol. 42.

[19] I. Hiskens, "Stability of hybrid limit cycles: application to the compass gait biped robot," in Proc. of the 40th IEEE Conference on Decision and Control, Orlando, FL, December 2001, pp. 774-779.

[20] Y. Hürmüzlü and D. Marghitu, "Rigid body collisions of planar kinematic chains with multiple contact points," International Journal of Robotics Research, vol. 13, no. 1, pp. 82-92, 1994.

[21] A. Isidori, Nonlinear Control Systems: An Introduction, 3rd ed. Berlin: Springer-Verlag, 1995.

[22] W. P. Johnson, “The curious history of faá di bruno's formula," American Mathematical Monthly, vol. 109, pp. 217-234, March 2002.

[23] H. Khalil, Nonlinear Systems - 2nd Edition. Upper Saddle River: Prentice Hall, 1996.

[24] B. Morris and J. W. Grizzle, "A restricted Poincaré map for determining exponentially stable periodic orbits in systems 
with impulse effects: Application to bipedal robots," in Proc. of IEEE 2005 Conference on Decision and Control, 2005, pp. 4199-4206.

[25] _ - "Hybrid invariance in bipedal robots with series compliant actuators," in Proc. of IEEE 2006 Conference on Decision and Control, 2006, pp. 4793-4800.

[26] S. Nersesov, V. Chellaboina, and W. Haddad, "A generalization of Poincaré's theorem to hybrid and impulsive dynamical systems," Int. J. Hybrid Systems, vol. 2, pp. 35-51, 2002.

[27] T. Parker and L. Chua, Practical Numerical Algorithms for Chaotic Systems. New York: Springer-Verlag, 1989.

[28] M. Reyhanoglu, A. van der Schaft, N. McClamroch, and I. Kolmanovsky, "Dynamics and control of a class of underactuated mechanical systems," IEEE Transactions on Automatic Control, vol. 44, no. 9, pp. 1663-1671, 1999.

[29] A. V. Roup, D. S. Bernstein, S. G. Nersesov, W. M. Haddad, and V. Chellaboina, "Limit cycle analysis of the verge and foliot clock escapement using impulsive differential equations and Poincare maps," Int. J. Control, vol. 76, no. 17, pp. 1685-1698, 2003.

[30] M. Spong, "Modeling and control of elastic joint robots," ASME Journal of Dynamic Systems, Measurement, and Control, vol. 109, pp. 310-319, Dec. 1987.

[31] — " "Adaptive control of flexible joint manipulators," Systems \& Control Letters, vol. 13, pp. 15-21, 1989.

[32] — " "Energy based control of a class of underactuated mechanical systems," in Proc. of IFAC World Congress, San Francisco, CA, 1996, pp. 431-435.

[33] E. Westervelt, J. W. Grizzle, and D. E. Koditschek, "Zero dynamics of planar biped walkers with one degree of under actuation," in IFAC 2002, Bareclona, Spain, July 2002.

[34] _ _ "Hybrid zero dynamics of planar biped walkers," IEEE Transactions on Automatic Control, vol. 48, no. 1, pp. 42-56, January 2003.

[35] E. R. Westervelt and J. W. Grizzle, "Design of asymptotically stable walking for a 5-link planar biped walker via optimization," in Proc. of the 2002 IEEE International Conference on Robotics and Automation, Washington, D.C., 2002, pp. 3117-3122.

[36] E. R. Westervelt, J. W. Grizzle, C. Chevallereau, J.-H. Choi, and B. Morris, Feedback Control of Dynamic Bipedal Robot Locomotion. Taylor \& Francis/CRC Press, 2007.

[37] E. R. Westervelt, B. Morris, and K. D. Farrell, "Analysis results and tools for the control of planar bipedal gaits using hybrid zero dynamics," Accepted for publication in the IEEE Journal of Autonomous Robots, 2007.

[38] H. Ye, A. Michel, and L. Hou, "Stability theory for hybrid dynamical systems," IEEE Transactions on Automatic Control, vol. 43, no. 4, pp. 461-474, April 1998.

\section{APPENDIX}

\section{A. Proof of Theorem 1 and its Corollaries}

Proof of Theorem 1: The (local) coordinate transform $\Gamma$ represents elements of the the submanifold $\mathcal{S} \cap \mathcal{Z}$ in preferred coordinates, so that ${ }^{13}$ (i) for any $\operatorname{point}^{14} x \in \mathcal{S} \cap \mathcal{Z} \cap U, \Gamma_{2}(x)=0$,

\footnotetext{
${ }^{13}$ Facts (i) and (ii) are properties easily derived from the definition of preferred coordinates in [8, p. 76].

${ }^{14}$ By definition, the domain $U$ of $\Gamma$ is a subset of $\mathcal{S}$ and thus $(U \cap \mathcal{S})=U$. To emphasize this fact, we prefer to designate the domain of $\Gamma$ as $(U \cap \mathcal{S})$.
} 
and (ii) for any point $x \in \mathcal{S} \cap U, \Gamma^{-1}\left(\Gamma_{1}(x), 0\right) \in \mathcal{S} \cap \mathcal{Z} \cap U$. Similarly, the coordinate transform $\Psi$ represents elements of $\mathcal{Z}$ in preferred coordinates: (i) for any $x \in \mathcal{Z} \cap V, \Psi_{2}(x)=0$, and (ii) for any point $x \in V, \Psi^{-1}\left(\Psi_{1}(x), 0\right) \in \mathcal{Z} \cap V$. The coordinate transforms $\Gamma$ and $\Psi$ must exist by virtue of the fact that $\mathcal{S} \cap \mathcal{Z}$ and $\mathcal{Z}$ are embedded submanifolds. Conditions H1.2) and the transversality portion of H1.3) are sufficient conditions under which the return map is differentiable at the point $x^{*}$. Let $\hat{P}_{1}(z, \eta)=\Gamma_{1} \circ P \circ \Gamma^{-1}(z, \eta)$ and $\hat{P}_{2}(z, \eta)=\Gamma_{2} \circ P \circ \Gamma^{-1}(z, \eta)$ so that the Jacobian of the return map can be written as

$$
\mathcal{D} \hat{P}(z, \eta)=\left[\begin{array}{ll}
\mathcal{D}_{1} \hat{P}_{1}(z, \eta) & \mathcal{D}_{2} \hat{P}_{1}(z, \eta) \\
\mathcal{D}_{1} \hat{P}_{2}(z, \eta) & \mathcal{D}_{2} \hat{P}_{2}(z, \eta)
\end{array}\right],
$$

which, when evaluated at $\left(z^{*}, \eta^{*}\right)=\Gamma\left(x^{*}\right)$, reduces to (9). By H1.3), the fixed point $x^{*}$ lies within $\mathcal{Z}$, and as a consequence of property (i) of $\Gamma, \eta^{*}=\Gamma_{2}\left(x^{*}\right)=0$. By the definition of $\hat{P}_{1}$ above and $\rho$ in $(8), \hat{P}_{1}(z, 0) \equiv \rho(z)$, implying that $\mathcal{D}_{1} \hat{P}_{1}\left(z^{*}, \eta^{*}\right)=\mathcal{D} \rho\left(z^{*}\right)$ and proving the form of the upper left block of (9). The hypothesis on hybrid invariance, H1.1), is a sufficient condition for (7) and (by property (i) of $\Gamma$ ) implies that $\hat{P}_{2}(z, 0)=0$ at all points $(z, 0)$ of its domain. Differentiation with respect to the $z$ coordinates gives $\mathcal{D}_{1} \hat{P}_{2}\left(z^{*}, \eta^{*}\right)=0$, which is the lower left block of (9). Applying the chain rule ${ }^{15}$ to the alternative form of the return map gives,

$$
\begin{aligned}
\mathcal{D}_{2} \hat{P}_{2}\left(z^{*}, \eta^{*}\right)= & D_{2}\left(\Gamma_{2} \circ \phi_{T_{I}} \circ \Delta \circ \Gamma^{-1}\right)\left(z^{*}, \eta^{*}\right) \\
= & \mathcal{D}_{2}\left(\left(\Gamma_{2} \circ \phi_{T_{I}} \circ \Psi^{-1}\right) \circ\left(\Psi \circ \Delta \circ \Gamma^{-1}\right)\right)\left(z^{*}, \eta^{*}\right) \\
= & \mathcal{D}_{1}\left(\Gamma_{2} \circ \phi_{T_{I}} \circ \Psi^{-1}\right)\left(\bar{z}^{*}, \bar{\eta}^{*}\right) \mathcal{D}_{2}\left(\Psi_{1} \circ \Delta \circ \Gamma^{-1}\right)\left(z^{*}, \eta^{*}\right) \\
& \quad+\mathcal{D}_{2}\left(\Gamma_{2} \circ \phi_{T_{I}} \circ \Psi^{-1}\right)\left(\bar{z}^{*}, \bar{\eta}^{*}\right) \mathcal{D}_{2}\left(\Psi_{2} \circ \Delta \circ \Gamma^{-1}\right)\left(z^{*}, \eta^{*}\right)
\end{aligned}
$$

Forward invariance of $\mathcal{Z}$ implies that $\mathcal{D}_{1}\left(\Gamma_{2} \circ \phi_{T_{I}} \circ \Psi^{-1}\right)\left(\bar{z}^{*}, \bar{\eta}^{*}\right)=0$, leading to the expression $\mathcal{D}_{2} \hat{P}_{2}\left(z^{*}, \eta^{*}\right)=S_{\phi_{T_{I}}}\left(\bar{z}^{*}, \bar{\eta}^{*}\right) S_{\Delta}\left(z^{*}, \eta^{*}\right)$, which completes the derivation of the form (9).

Lemma 12: Suppose that for some $r>0, F: B_{r}(0) \rightarrow \mathbb{R}^{n}$ satisfies

a) $\exists L<\infty$ such that $\forall x \in B_{r}(0),\|F(x)\| \leq L\|x\|$; and

b) $F$ is continuously differentiable at every point in $B_{r}(0)$.

Then, $\|\partial F(0) / \partial x\|_{i} \leq L$ where $\|\cdot\|_{i}$ is the induced norm.

\footnotetext{
${ }^{15}$ For any differentiable functions $F_{1}: \mathbb{R}^{m} \times \mathbb{R}^{n} \rightarrow \mathbb{R}^{u}, F_{2}: \mathbb{R}^{m} \times \mathbb{R}^{n} \rightarrow \mathbb{R}^{v}, F\left(x_{1}, x_{2}\right)=\left(F_{1}\left(x_{1}, x_{2}\right), F_{2}\left(x_{1}, x_{2}\right)\right)$, and $G: \mathbb{R}^{u} \times \mathbb{R}^{v} \rightarrow \mathbb{R}^{p}$, application of the chain rule shows that $\mathcal{D}_{2}(G \circ F)\left(x_{1}, x_{2}\right)=D_{1} G \cdot D_{2} F_{1}+D_{2} G \cdot D_{2} F_{2}$.
} 
Proof: By (b) and Taylor's theorem, $F(x)=F(0)+(\partial F(0) / \partial x) x+R(x)$ where $\lim _{x \rightarrow 0}\|R(x)\| /\|x\|=0$. By (a), $F(0)=0$ and $\|(\partial F(0) / \partial x) x+R(x)\|=\|F(x)\| \leq L\|x\|$. By compactness of closed unit balls in $\mathbb{R}^{n}$, there exists $\bar{x}$ such that $\|(\partial F(0) / \partial x) \bar{x}\|=\|(\partial F(0) / \partial x)\|_{i}$ and $\|\bar{x}\|=1$. Letting $x=\delta \bar{x}$, for any value of $\delta>0,\|(\partial F(0) / \partial x)(\delta \bar{x} /\|\delta \bar{x}\|)+(R(\delta \bar{x}) /\|\delta \bar{x}\|)\|=$ $\|(\partial F(0) / \partial x) \bar{x}+(R(\delta \bar{x}) /\|\delta \bar{x}\|)\| \leq L$. It follows that $\lim _{\delta \searrow 0}\|(\partial F(0) / \partial x) \bar{x}+(R(\delta \bar{x}) /\|\delta \bar{x}\|)\|=$ $\|(\partial F(0) / \partial x)\|_{i}$ and hence $\|(\partial F(0) / \partial x)\|_{i} \leq L$.

Remark 13: Any parameterized function $F^{\epsilon}: B_{r(\epsilon)}(0) \rightarrow \mathbb{R}^{n}, \quad r(\epsilon)>0$ that satisfies

a) for each $\epsilon>0, F^{\epsilon}(0)=0$;

b) for each $\epsilon>0$, there exists $\delta(\epsilon)>0$ such that $F^{\epsilon}(x)$ is continuously differentiable on $B_{\delta(\epsilon)}(0) ;$ and

c) $\left\|F^{\epsilon}(x)\right\| \leq L(\epsilon)\|x\|$ with $\lim _{\epsilon \backslash 0} L(\epsilon)=0$,

must (by Lemma 12) have the property that $\lim _{\epsilon \backslash 0}\left\|\frac{\partial F^{\epsilon}}{\partial x}(0)\right\|_{i}=0$.

Proof of Corollary 2: The first claim of the corollary is trivial to prove: the system $\Sigma^{c l, \epsilon}$ restricted to the hybrid invariant manifold $\mathcal{Z}$ is independent of $\epsilon$. By $\mathrm{H} 2.1$ ) and $\mathrm{H} 2.2$ ), the manifold $\mathcal{Z}$ is independent of $\epsilon$ and so is the vector field $\left.f^{\epsilon}\right|_{\mathcal{Z}}$. For the second claim of the corollary, the Method of Poincaré Sections (Theorem 0) is used to establish a relationship between the eigenvalues of the Jacobian of a Poincare map and the stability of the underlying orbit. Because they are unaffected by coordinate transforms, the eigenvalues of $\mathcal{D} P^{\epsilon}\left(x^{*}\right)$ are equal to the eigenvalues of $\mathcal{D} \hat{P}^{\epsilon}\left(z^{*}, \eta^{*}\right)$. As shown in Theorem 1, the matrix $\mathcal{D} \hat{P}^{\epsilon}\left(z^{*}, \eta^{*}\right)$ is block upper triangular for all values of $\epsilon$, and therefore

$$
\operatorname{eig}\left(D P^{\epsilon}\left(x^{*}\right)\right)=\operatorname{eig}\left(\mathcal{D} \rho\left(z^{*}\right)\right) \cup \operatorname{eig}\left(S_{\phi_{T_{I}}}^{\epsilon}\left(\bar{z}^{*}, \bar{\eta}^{*}\right) S_{\Delta}\left(z^{*}, \eta^{*}\right)\right)
$$

Assume that $\lim _{\epsilon \backslash 0} S_{\phi_{T_{I}}}^{\epsilon}\left(\bar{z}^{*}, \bar{\eta}^{*}\right)=0$ (a fact to be proven below). In this case, for $\epsilon$ sufficiently small, the maximum eigenvalue of $D P^{\epsilon}\left(x^{*}\right)$ is equal to the maximum eigenvalue of $\mathcal{D} \rho\left(z^{*}\right)$, and by the Method of Poincaré Sections, the orbit $\mathcal{O}$ is exponentially stable in the full system $\Sigma^{c l, \epsilon}$ if and only if the same orbit is exponentially stable in the restricted system $\left.\Sigma^{c l}\right|_{\mathcal{Z}}$.

To show that $\lim _{\epsilon \backslash 0} S_{\phi_{T_{I}}}^{\epsilon}\left(\bar{z}^{*}, \bar{\eta}^{*}\right)=0$, invoke the convergence property of $\left.\mathrm{H} 2.3\right)$ in the application of Taylor's theorem in Lemma 12. To start, note that the function $\Gamma$ is differentiable and therefore locally Lipschitz continuous. That is, there exists $L_{\Gamma}>0$ such that for all $x$ in an 
open neighborhood of $U \cap \mathcal{S}$ containing the point $x^{*}$,

$$
\begin{aligned}
\operatorname{dist}(x, \mathcal{Z})=\inf _{y \in \mathcal{Z}}\|x-y\| & \geq \inf _{y \in \mathcal{Z}} \frac{1}{L_{\Gamma}}\|\Gamma(x)-\Gamma(y)\| \\
& =\inf _{y \in \mathcal{Z}} \frac{1}{L_{\Gamma}}\left\|\left(\Gamma_{1}(x), \Gamma_{2}(x)\right)-\left(\Gamma_{1}(y), 0\right)\right\| .
\end{aligned}
$$

The last line in the above is obtained using property (i) of the preferred coordinates given by $\Gamma$ (as used in the proof of Theorem 1). By property (ii) of $\Gamma, \forall x \in U \cap \mathcal{S}, \Gamma^{-1}\left(\Gamma_{1}(x), 0\right) \in U \cap \mathcal{S} \cap \mathcal{Z}$. Stated differently, $\forall x \in U \cap \mathcal{S}, \exists y \in U \cap \mathcal{S} \cap Z$ such that $\Gamma_{1}(x)=\Gamma_{1}(y)$. Applying this to the last line of (22) shows that

$$
\operatorname{dist}(x, \mathcal{Z}) \geq \frac{1}{L_{\Gamma}}\left\|\left(\Gamma_{1}(x), \Gamma_{2}(x)\right)-\left(\Gamma_{1}(x), 0\right)\right\|=\frac{1}{L_{\Gamma}}\left\|\Gamma_{2}(x)\right\|
$$

Next, by the triangle inequality, for any $x_{0}$ in an open neighborhood of $V$ containing $\Delta\left(x^{*}\right)$,

$$
\operatorname{dist}\left(x_{0}, \mathcal{Z}\right) \leq\left\|x_{0}-\Psi^{-1}\left(\Psi_{1}\left(x_{0}\right), 0\right)\right\|
$$

Writing $x_{0}$ as the identity $x_{0}=\Psi^{-1}\left(\Psi_{1}\left(x_{0}\right), \Psi_{2}\left(x_{0}\right)\right)$ gives,

$$
\begin{aligned}
\operatorname{dist}\left(x_{0}, \mathcal{Z}\right) & \leq\left\|\Psi^{-1}\left(\Psi_{1}\left(x_{0}\right), \Psi_{2}\left(x_{0}\right)\right)-\Psi^{-1}\left(\Psi_{1}\left(x_{0}\right), 0\right)\right\| \\
& \leq L_{\Psi^{-1}}\left\|\left(\Psi_{1}\left(x_{0}\right), \Psi_{2}\left(x_{0}\right)\right)-\left(\Psi_{1}\left(x_{0}\right), 0\right)\right\|=L_{\Psi^{-1}}\left\|\Psi_{2}\left(x_{0}\right)\right\|,
\end{aligned}
$$

for some finite $L_{\Psi^{-1}}>0$ (as $\Psi^{-1}$ is also locally Lipschitz).

Recall the following facts: $\Delta\left(x^{*}\right)$ lies within the open set $V$; for every $\epsilon>0, \phi_{T_{I}}^{\epsilon}\left(\Delta\left(x^{*}\right)\right)=x^{*}$ lies within the open set $U \cap S$; and for every $\epsilon>0, \Delta\left(x^{*}\right)$ lies within the open set $B_{\delta}\left(\Delta\left(x^{*}\right)\right)$ for $\delta$ from H2.3). The function $\Delta$ is continuous, as is $\phi_{T_{I}^{\epsilon}}^{\epsilon}$ for each value of $\epsilon>0$. Thus, for every $\epsilon>0$ there exists $\mu>0$ such that $B_{\mu}\left(\Delta\left(x^{*}\right)\right) \subset V, \phi_{T_{I}}^{\epsilon}\left(B_{\mu}\left(\Delta\left(x^{*}\right)\right)\right) \subset(U \cap \mathcal{S})$, and $\mu<\delta$. Together, (23), (25), and H2.3) imply that $\forall x_{0} \in B_{\mu}\left(\Delta\left(x^{*}\right)\right)$

$$
\frac{1}{L_{\Gamma}}\left\|\Gamma_{2} \circ \phi_{T_{I}}^{\epsilon}\left(x_{0}\right)\right\| \leq \operatorname{dist}\left(\phi_{T_{I}}^{\epsilon}\left(x_{0}\right), \mathcal{Z}\right) \leq K(\epsilon) \operatorname{dist}\left(x_{0}, \mathcal{Z}\right) \leq K(\epsilon) L_{\Psi^{-1}}\left\|\Psi_{2}\left(x_{0}\right)\right\|
$$

Setting $(\bar{z}, \bar{\eta})=\Psi\left(x_{0}\right)$ leads to $\left\|\Gamma_{2} \circ \phi_{T_{I}^{\epsilon}}^{\epsilon} \circ \Psi^{-1}(\bar{z}, \bar{\eta})\right\| \leq L_{\Gamma} L_{\Psi^{-1}} K(\epsilon)\|\bar{\eta}\|$. The periodic orbit $\mathcal{O}$ is contained in $\mathcal{Z}$ and thus for all $\epsilon>0, \eta^{*}=\left(\Gamma_{2} \circ \phi_{T_{I}^{\epsilon}}^{\epsilon} \circ \Psi^{-1}\right)\left(\bar{z}^{*}, \bar{\eta}^{*}\right)=0$. Thus, the function $\left(\Gamma_{2} \circ \phi_{T_{I}}^{\epsilon} \circ \Psi^{-1}\right)\left(\bar{z}^{*}, \bar{\eta}^{*}\right)$ meets the criteria of Lemma 12 and Remark 13, which imply that $\lim _{\epsilon \backslash 0} D_{2}\left(\Gamma_{2} \circ \phi_{T_{I}}^{\epsilon} \circ \Psi^{-1}\right)\left(\bar{z}^{*}, \bar{\eta}^{*}\right)=0$, or, equivalently $\lim _{\epsilon}{ }_{0} S_{\phi_{T_{I}}^{\epsilon}}^{\epsilon}\left(\bar{z}^{*}, \bar{\eta}^{*}\right)=0$, which was to be shown.

Proof of Corollary 4: Forward invariance and the submanifold property of $\mathcal{Z}$ follow immediately from applying the general results of [21, Ch.5] to the drift and control vector fields of 
$\Sigma^{o l}$. Condition H2.1) of Corollary 2 is trivially satisfied because $\epsilon$ does not participate in the definition of $\mathcal{Z}$. Similarly, the feedback (10) is independent of $\epsilon$ on the manifold $\mathcal{Z}$ and therefore the closed-loop flow $f(x)+g(x) u^{\epsilon}(x)$ when restricted to $\mathcal{Z}$ is independent of $\epsilon$.

Under the feedback (10), the manifold $\mathcal{Z}$ is exponentially attractive with $\epsilon$-dependant convergence parameters $c$ and $\gamma$ satisfying ${ }^{16} \forall 0 \leq t<T_{I}^{\epsilon}\left(x_{0}\right) \operatorname{dist}\left(\phi^{\epsilon}\left(t, x_{0}\right), \mathcal{Z}\right) \leq c(\epsilon) e^{-\gamma(\epsilon) t} \operatorname{dist}\left(x_{0}, \mathcal{Z}\right)$ and $\lim _{\epsilon \backslash 0} c(\epsilon) e^{-\gamma(\epsilon)}=0$. For a given $\epsilon>0$, choose $\delta>0$ such that $\phi_{T_{I}}{ }^{\epsilon}\left(x_{0}\right)$ exists for all $x_{0}$ in the closed ball $\bar{B}_{\delta}\left(\Delta\left(x^{*}\right)\right)$. On this compact set, the differentiable function $T_{I}^{\epsilon}(x)$ achieves a minimum value. If necessary, further restrict $\delta$ so that this minimum value is strictly greater than one half of the period $t^{*}$ of the orbit $\mathcal{O}$. Then for the chosen $\epsilon$ and corresponding $\delta$, each $x_{0}$ in the open ball $B_{\delta}\left(\Delta\left(x^{*}\right)\right)$ satisfies $\operatorname{dist}\left(\phi_{T_{I}}{ }^{\epsilon}\left(x_{0}\right), \mathcal{Z}\right) \leq c(\epsilon) e^{-\gamma(\epsilon) T_{I}^{\epsilon}\left(x_{0}\right)} \operatorname{dist}\left(x_{0}, \mathcal{Z}\right) \leq$ $c(\epsilon) e^{-\gamma(\epsilon) \frac{1}{2} t^{*}} \operatorname{dist}\left(x_{0}, \mathcal{Z}\right)$. Define $K(\epsilon)=c(\epsilon) e^{-\gamma(\epsilon) \frac{1}{2} t^{*}}$. Then for each value of $\epsilon>0$ there exists $\delta>0$ such that for all $x_{0} \in B_{\delta}\left(\Delta\left(x^{*}\right)\right)$, $\operatorname{dist}\left(\phi_{T_{I}}^{\epsilon}\left(x_{0}\right), \mathcal{Z}\right) \leq K(\epsilon) \operatorname{dist}\left(x_{0}, \mathcal{Z}\right)$, with $\lim _{\epsilon \backslash 0} K(\epsilon)=0$. Thus Hypothesis H2.3) of Corollary 2 is satisfied, completing the proof of Corollary 4.

\section{B. Proof of Theorem 7}

This section constructs the open-loop deadbeat hybrid extension $\Sigma_{e}^{o l, \lambda}=\left(\mathcal{X}_{e}, \mathcal{S}_{e}, \emptyset, \mathcal{U}, \Delta_{e}^{c l, \lambda}, f_{e}, g_{e}\right)$ and output function $y_{e}=h_{e}\left(x_{e}\right)$ used in the proof of Theorem 7. A proof of the theorem is then given. To begin the construction of $y_{e}=h_{e}\left(x_{e}\right)$, choose any function $B: \mathbb{R} \times \mathbb{R}^{m k} \rightarrow \mathbb{R}^{m}$ satisfying the properties ${ }^{17}$

i) for any $b=\left(b_{0}, \ldots, b_{k-1}\right), b_{0}, \ldots, b_{k-1} \in \mathbb{R}^{m}$

$$
\left.B(s, b)\right|_{s=0}=b_{0},\left.\frac{\partial}{\partial s} B(s, b)\right|_{s=0}=b_{1}, \ldots,\left.\frac{\partial^{k-1}}{\partial s^{k-1}} B(s, b)\right|_{s=0}=b_{k-1} ;
$$

ii) for any $b=\left(b_{0}, \ldots, b_{k-1}\right), b_{0}, \ldots, b_{k-1} \in \mathbb{R}^{m}$

$$
\left.B(s, b)\right|_{s=1}=0,\left.\frac{\partial}{\partial s} B(s, b)\right|_{s=1}=0, \ldots,\left.\frac{\partial^{k}}{\partial s^{k}} B(s, b)\right|_{s=1}=0 ;
$$

iii) $\forall s \in \mathbb{R}, B(s, 0) \equiv 0$;

iv) $\forall b \in \mathbb{R}^{m k}$, the function $B(s, b)$ is $C^{k+1}$ in $s$; and

v) $\forall s \in \mathbb{R}$, each of the functions $B(s, b), \frac{\partial}{\partial s} B(s, b), \ldots, \frac{\partial^{k}}{\partial s^{k}} B(s, b)$ is continuous in $b$.

\footnotetext{
${ }^{16}$ As specified earlier, the initialization time for $\phi\left(t, x_{0}\right)$ is always assumed to be $t_{0}=0$.

${ }^{17}$ That is, $B$ is a vector-valued $C^{k+1}$ spline.
} 
Continuing, define a function $s: \mathcal{X} \times \mathbb{R} \rightarrow \mathbb{R}$ as $s\left(x, s_{0}\right)=2 \tau(x)+s_{0}$, and note that by monotonicity of $\tau(x)$ (Hypothesis H7.3) $s\left(x, s_{0}\right)$ will be strictly monotonically increasing (that is $L_{f} s\left(x, s_{0}\right)=2 L_{f}(x) \tau>0$ on $\mathcal{O}$ ) for any choice of $s_{0}$. Define the parameter vector $\alpha=$ $\left(b, s_{0}\right) \in \mathbb{R}^{m k+1}$ for $b \in \mathbb{R}^{m k}$ and $s_{0} \in \mathbb{R}$, and designate an extended state vector as $x_{e}=(x, \alpha)$. With this notation, the constructed output function is written as

$$
h_{e}\left(x_{e}\right)= \begin{cases}h(x)+B\left(s\left(x, s_{0}\right), b\right) & \text { for } s\left(x, s_{0}\right)<1 \\ h(x) & \text { else. }\end{cases}
$$

Motivated by the parameter vector of the constructed output (27), let $\mathcal{A}=\mathbb{R}^{m k+1}$. In general, there are uncountably many parameter update functions that could be constructed to satisfy Theorem 7. One family of such updates is indexed by a scalar $\lambda \in \mathbb{R}$ with

$$
v^{\lambda}\left(x^{-}\right)=\left(b_{0}^{\lambda}\left(x^{-}\right), \ldots, b_{k-1}^{\lambda}\left(x^{-}\right), s_{0}\left(x^{-}\right)\right)
$$

where $s_{0}\left(x^{-}\right)=-2 \tau\left(x^{+}\right), b_{0}^{\lambda}\left(x^{-}\right)=\lambda h\left(x^{-}\right)-h\left(x^{+}\right)$, and

$$
b_{n}^{\lambda}\left(x^{-}\right)=\left(2 L_{f} \tau\left(x^{+}\right)\right)^{-n}\left(-L_{f}^{n} h\left(x^{+}\right)+\lambda L_{f}^{n} h\left(x^{-}\right)-\mathcal{R}_{n}^{(1)}\left(x^{+}, b_{0}\left(x^{-}\right), \ldots, b_{n-1}\left(x^{-}\right)\right)\right)
$$

for $x^{-} \in \mathcal{S}, x^{+}=\Delta\left(x^{-}\right)$, and $1 \leq n \leq k-1$. The term $\mathcal{R}_{n}^{(1)}\left(x^{+}, b_{0}, \ldots, b_{n-1}\right)$ will be defined shortly, following Remark 14. Letting $\mathcal{X}_{e}=\mathcal{X} \times \mathcal{A}, \mathcal{S}_{e}=\mathcal{S} \times \mathcal{A}, \Delta_{e}^{c l, \lambda}\left(x_{e}\right)=\left(\Delta(x), v^{\lambda}(x)\right)$, $x_{e}=(x, \alpha), f_{e}\left(x_{e}\right)=(f(x), 0)$, and $g_{e}\left(x_{e}\right)=(g(x), 0)$ leads to the final construction of the open-loop deadbeat hybrid extension, $\Sigma_{e}^{o l, \lambda}=\left(\mathcal{X}_{e}, \mathcal{S}_{e}, \emptyset, \mathcal{U}, \Delta_{e}^{c l, \lambda}, f_{e}, g_{e}\right)$.

Remark 14: For the composition $B\left(s\left(x, s_{0}\right), b\right)$, Faá di Bruno's formula [22] for the $n^{\text {th }}$ partial derivative generalizes ${ }^{18}$ to a formula for the $n^{\text {th }}$ Lie derivative

$$
L_{f}^{n} B\left(s\left(x, s_{0}\right), b\right)=\sum_{\mathcal{J}_{n}} \frac{n !}{j_{1} ! j_{2} ! \ldots j_{n} !} \frac{\partial^{j} B\left(s\left(x, s_{0}\right), b\right)}{\partial s^{j}} \prod_{i=1}^{n}\left(\frac{L_{f}^{i} s\left(x, s_{0}\right)}{i !}\right)^{j_{i}}
$$

where $j=j_{1}+\cdots+j_{n}$ and the summation is over the set $\mathcal{J}_{n}$ of all n-tuples of nonnegative integer values $\left(j_{1}, \ldots, j_{n}\right)$ satisfying $j_{1}+2 j_{2}+\cdots+n j_{n}=n$.

For use in (29), let $\mathcal{R}_{n}^{(1)}(x, \alpha)$ represent the summation of (30) over the index set $\mathcal{J}_{n}^{(1)}=$ $\mathcal{J}_{n} \backslash\left\{(n, 0, \ldots, 0\}\right.$, so that with $\alpha=\left(b, s_{0}\right)$ and $x_{e}=(x, \alpha)$,

$$
L_{f_{e}}^{n} h_{e}\left(x_{e}\right)=L_{f}^{n} h(x)+\mathcal{R}_{n}^{(1)}(x, \alpha)+\frac{\partial^{n} B\left(s\left(x, s_{0}\right), b\right)}{\partial s^{n}}\left(L_{f} s\left(x, s_{0}\right)\right)^{n} .
$$

\footnotetext{
${ }^{18}$ This generalization is only possible because the function $s$ is scalar-valued.
} 
By property i) of $B$, when $x$ and $s_{0}$ are such that $s\left(x, s_{0}\right)=0$, the value of $\mathcal{R}_{n}^{(1)}(x, \alpha)$ is dependent only on $x$ and the parameters $b_{0}, \ldots, b_{n-1}$, and the notation $\mathcal{R}_{n}^{(1)}\left(x, b_{0}, \ldots, b_{n-1}\right)$ becomes appropriate. For use in the proof of Lemma 15 , let $\mathcal{R}_{n}^{(2)}(x, \alpha)$ represent the summation of (30) over the index set $\mathcal{J}_{n}^{(2)}=\mathcal{J}_{n} \backslash\{(0, \ldots, 0,1)\}$, so that with $\alpha=\left(b, s_{0}\right)$ and $x_{e}=(x, \alpha)$,

$$
L_{f_{e}}^{n} h_{e}\left(x_{e}\right)=L_{f}^{n} h(x)+\mathcal{R}_{n}^{(2)}(x, \alpha)+\frac{\partial B\left(s\left(x, s_{0}\right), b\right)}{\partial s} L_{f}^{n} s\left(x, s_{0}\right) .
$$

Lemma 15: The output $h_{e}\left(x_{e}\right)$ of (27) has uniform vector relative degree $k$ for all $x_{e}$ in an open neighborhood of the $\mathcal{O}_{e}$, which is the trivial lift of $\mathcal{O}$ into $\Sigma_{e}^{o l}$.

Proof: For all $x_{e} \in \mathcal{X}_{e}, 0 \leq n \leq k-1$

$$
L_{f_{e}}^{n} h_{e}\left(x_{e}\right)= \begin{cases}L_{f}^{n} h(x)+L_{f}^{n} B\left(s\left(x, s_{0}\right), b\right) & \text { for } s\left(x, s_{0}\right)<1 \\ L_{f}^{n} h(x) & \text { else. }\end{cases}
$$

By H7.1), the claim of the Lemma is trivial for all $x_{e} \in \mathcal{X}_{e}$ for which $s\left(x, s_{0}\right)>1$. Using the term $\mathcal{R}_{n}^{(2)}(x, \alpha)$ developed after Remark 14, expand the first line of (33) to obtain that for all $x_{e}=(x, \alpha) \in \mathcal{X}_{e}$ such that $s\left(x, s_{0}\right)<1$, for $0 \leq n \leq k-1$,

$$
L_{f_{e}}^{n} h_{e}\left(x_{e}\right)=L_{f}^{n} h(x)+\mathcal{R}_{n}^{(2)}(x, \alpha)+\frac{\partial B\left(s\left(x, s_{0}\right), b\right)}{\partial s} L_{f}^{n} s\left(x, s_{0}\right),
$$

which is (32). Each additive term of $\mathcal{R}_{n}^{(2)}(x, \alpha)$ contains $L_{f}^{i} s\left(x, s_{0}\right)$ for some $0 \leq i \leq n-1$. From its definition, the function $s\left(x, s_{0}\right)$ satisfies the property that $\forall x \in \mathcal{X}, \forall s_{0} \in \mathbb{R}$ and $0 \leq n \leq k-1$, $L_{g} L_{f}^{n} s\left(x, s_{0}\right)=2 L_{g} L_{f}^{n} \tau(x)$. And, by H7.3), $L_{g} L_{f}^{n} \tau(x)=0$ for $0 \leq n \leq k-2$. With omitted chain-rule calculations left to the reader, this further implies that for all $x_{e}=(x, \alpha)=\left(x, b, s_{0}\right) \in$ $\mathcal{X}_{e}$ such that $s\left(x, s_{0}\right)<1,0 \leq n \leq k-2$, it holds that $L_{g} \mathcal{R}_{n}^{(2)}\left(x, b, s_{0}\right)=0$. Accordingly, for all $x_{e}=(x, \alpha) \in \mathcal{X}_{e}$ such that $s\left(x, s_{0}\right)<1$, for $0 \leq n \leq k-2, L_{g_{e}} L_{f_{e}}^{n} h_{e}\left(x_{e}\right)=0$, which is part i) of the definition uniform vector relative degree. In the case of $n=k-1$, (34) simplifies to

$$
L_{g_{e}} L_{f_{e}}^{k-1} h_{e}\left(x_{e}\right)=L_{g} L_{f}^{k-1} h(x)+L_{g}\left(\frac{\partial B\left(s\left(x, s_{0}\right), b\right)}{\partial s} L_{f}^{k-1} s\left(x, s_{0}\right)\right)
$$

giving the decoupling matrix as

$$
L_{g_{e}} L_{f_{e}}^{k-1} h_{e}\left(x_{e}\right)=L_{g} L_{f}^{k-1} h(x)+\frac{\partial B\left(s\left(x, s_{0}\right), b\right)}{\partial s} L_{g} L_{f}^{k-1} s\left(x, s_{0}\right) .
$$

Applying the Sherman-Morrison-Woodbury formula [6], the decoupling matrix is invertible at each point $x_{e}=\left(x, b, s_{0}\right) \in \mathcal{X}_{e}$ where the continuous scalar function

$$
1+L_{g} L_{f}^{k-1} s\left(x, s_{0}\right)\left(L_{g} L_{f}^{k-1} h(x)\right)^{-1} \frac{\partial B\left(s\left(x, s_{0}\right), b\right)}{\partial s}
$$


is nonzero. Along $\mathcal{O}_{e}$, the trivial lift of $\mathcal{O}$, the parameter $b$ takes a value of $0 \in \mathbb{R}^{m k}$ and thus by property iii) of $B$, for all $\left(x, b, s_{0}\right) \in \mathcal{O}_{e}, \partial B\left(s\left(x, s_{0}\right), b\right) / \partial s=0$. As a result the function in (36) has a constant value of 1 on the orbit $\mathcal{O}_{e}$. Because (36) is continuous and nonzero on $\mathcal{O}_{e}$, it must be nonzero in an open neighborhood of $\mathcal{O}_{e}$. Equivalently, the decoupling matrix $L_{g_{e}} L_{f_{e}}^{k-1} h_{e}\left(x_{e}\right)$ is invertible in an open neighborhood of $\mathcal{O}_{e}$, which fulfills part ii) of the definition of uniform vector relative degree.

Proof of Theorem 7: By Lemma 15, the parameterized extension, $\Sigma_{e}^{o l, \lambda}$ and output function $h_{e}$ together fulfill H4.1) of Corollary 4. Hypothesis H7.1) of Theorem 7 implies that H4.2) of Corollary 4 is true - indeed every point on the trivial lift $\mathcal{O}_{e}$ meets this condition. Hypothesis H7.2) of Theorem 7 implies that the open-loop deadbeat hybrid extension meets H4.3). The manifold $\mathcal{Z}_{e}$ is impact invariant if and only for all $x_{e}^{-}=\left(x^{-}, \alpha^{-}\right) \in \mathcal{S}_{e} \cap \mathcal{Z}_{e}$,

$$
h_{e}\left(x^{+}, \alpha^{+}\right)=0, L_{f_{e}} h_{e}\left(x^{+}, \alpha^{+}\right)=0, \ldots, L_{f_{e}}^{k-1} h_{e}\left(x^{+}, \alpha^{+}\right)=0
$$

with $x^{+}=\Delta\left(x^{-}\right)$and $\alpha^{+}=v^{\lambda}\left(x^{-}\right)$. The above Lie derivatives can be expanded as in (31); $\forall x^{-} \in \mathcal{S}$ with $x^{+}=\Delta\left(x^{-}\right)$and $0 \leq n \leq k-1$,

$$
L_{f_{e}}^{n} h_{e}\left(x^{+}, \alpha\right)=L_{f}^{n} h\left(x^{+}\right)+\mathcal{R}_{n}^{(1)}\left(x^{+}, \alpha\right)+\frac{\partial^{n} B\left(s\left(x^{+}, s_{0}\right), b\right)}{\partial s^{n}}\left(L_{f} s\left(x^{+}, s_{0}\right)\right)^{n},
$$

for any $\alpha \in \mathcal{A}$. By the construction of $s, L_{f} s\left(x, s_{0}\right)=2 L_{f} \tau(x)$ (independent of the value of $s_{0}$ ). After the update of $s_{0}=-2 \tau\left(x^{+}\right)$, the value of $s\left(x^{+}, s_{0}\right)$ is necessarily zero. Using property i) of $B$, the $n=0$ case of (37) is simplified to $h_{e}\left(x^{+}, b, s_{0}\right)=h\left(x^{+}\right)+b_{0}$, and for $1 \leq n \leq k-1$, $L_{f_{e}}^{n} h_{e}\left(x^{+}, b, s_{0}\right)=L_{f}^{n} h\left(x^{+}\right)+b_{n}\left(2 L_{f} \tau\left(x^{+}\right)\right)^{n}+\mathcal{R}_{n}^{(1)}\left(x^{+}, b_{0}, \ldots, b_{n-1}\right)$. The parameter updates of (29) are derived by setting $L_{f_{e}}^{n} h_{e}\left(x^{+}, b, s_{0}\right)=\lambda L_{f}^{n} h\left(x^{-}\right)$and solving for $b_{n}$. In this way, impact invariance of $\mathcal{Z}_{e}$ is achieved by construction. Lastly, Hypothesis H7.1) and property iii) of $B$ imply that the orbit $\mathcal{O}_{e}$ is in $\mathcal{Z}_{e}$, which is the final claim of the theorem. 